\title{
Graph Minors and Minimum Degree
}

\author{
Gašper Fijavž \\ Faculty of Computer and Information Science \\ University of Ljubljana \\ Ljubljana, Slovenia \\ gasper.fijavz@fri.uni-lj.si \\ David R. Wood \\ Department of Mathematics and Statistics \\ The University of Melbourne \\ Melbourne, Australia \\ woodd@unimelb.edu.au
}

Submitted: Dec 7, 2008; Accepted: Oct 22, 2010; Published: Nov 5, 2010

Mathematics Subject Classifications: 05C83 graph minors

\begin{abstract}
Let $\mathcal{D}_{k}$ be the class of graphs for which every minor has minimum degree at most $k$. Then $\mathcal{D}_{k}$ is closed under taking minors. By the Robertson-Seymour graph minor theorem, $\mathcal{D}_{k}$ is characterised by a finite family of minor-minimal forbidden graphs, which we denote by $\widehat{\mathcal{D}}_{k}$. This paper discusses $\widehat{\mathcal{D}}_{k}$ and related topics. We obtain four main results:

1. We prove that every $(k+1)$-regular graph with less than $\frac{4}{3}(k+2)$ vertices is in $\widehat{\mathcal{D}}_{k}$, and this bound is best possible.

2. We characterise the graphs in $\widehat{\mathcal{D}}_{k+1}$ that can be obtained from a graph in $\widehat{\mathcal{D}}_{k}$ by adding one new vertex.

3. For $k \leqslant 3$ every graph in $\widehat{\mathcal{D}}_{k}$ is $(k+1)$-connected, but for large $k$, we exhibit graphs in $\widehat{\mathcal{D}}_{k}$ with connectivity 1 . In fact, we construct graphs in $\mathcal{D}_{k}$ with arbitrary block structure.

4. We characterise the complete multipartite graphs in $\widehat{\mathcal{D}}_{k}$, and prove analogous characterisations with minimum degree replaced by connectivity, treewidth, or pathwidth.
\end{abstract}

\footnotetext{
${ }^{1}$ D.W. is supported by a QEII Research Fellowship from the Australian Research Council. An extended abstract of this paper was published in: Proc. Topological 83 Geometric Graph Theory (TGGT '08), Electronic Notes in Discrete Mathematics 31:79-83, 2008.
} 


\section{Introduction}

The theory of graph minors developed by Robertson and Seymour [25] is one of the most important in graph theory influencing many branches of mathematics. Let $\mathcal{X}$ be a minorclosed class of graphs ${ }^{1}$. A graph $G$ is a minimal forbidden minor of $\mathcal{X}$ if $G$ is not in $\mathcal{X}$ but every proper minor of $G$ is in $\mathcal{X}$. Let $\widehat{\mathcal{X}}$ be the set of minimal forbidden minors of $\mathcal{X}$. By the graph minor theorem of Robertson and Seymour [25], $\widehat{\mathcal{X}}$ is a finite set. For various minor-closed classes the list of minimal forbidden minors is known. Most famously, if $\mathcal{P}$ is the class of planar graphs, then the Kuratowski-Wagner theorem states that $\widehat{\mathcal{P}}=\left\{K_{5}, K_{3,3}\right\}$. However, in general, determining the minimal forbidden minors for a particular minor-closed class is a challenging problem.

Let $\delta(G)$ be the minimum degree of a graph $G$. Let $\mathcal{D}_{k}$ be the class of graphs $G$ such that every minor of $G$ has minimum degree at most $k$. Then $\mathcal{D}_{k}$ is minor-closed. Let $\widehat{\mathcal{D}}_{k}$ be the set of minimal forbidden minors of $\mathcal{D}_{k}$. By the graph minor theorem, $\widehat{\mathcal{D}}_{k}$ is finite for each $k$. The structure of graphs in $\widehat{\mathcal{D}}_{k}$ is the focus of this paper. For small values of $k$, it is known that $\widehat{\mathcal{D}}_{0}=\left\{K_{2}\right\}$ and $\widehat{\mathcal{D}}_{1}=\left\{K_{3}\right\}$ and $\widehat{\mathcal{D}}_{2}=\left\{K_{4}\right\}$ and $\widehat{\mathcal{D}}_{3}=\left\{K_{5}, K_{2,2,2}\right\}$; see Section 2. Determining $\widehat{\mathcal{D}}_{4}$ is an open problem.

The majority of this paper studies the case of general $k$ rather than focusing on small values. Our first main result shows that, in some sense, there are many graphs in $\widehat{\mathcal{D}}_{k}$. In particular, every sufficiently small $(k+1)$-regular graph is in $\widehat{\mathcal{D}}_{k}$. This result is proved in Section 5 ,

Theorem 1.1. Every $(k+1)$-regular graph with less than $\frac{4}{3}(k+2)$ vertices is in $\widehat{\mathcal{D}}_{k}$. Moreover, for all $k \equiv 1(\bmod 3)$ there is a $(k+1)$-regular graph on $\frac{4}{3}(k+2)$ vertices that is not in $\widehat{\mathcal{D}}_{k}$.

Our second main result characterises the graphs in $\widehat{\mathcal{D}}_{k+1}$ that can be obtained from a graph in $\widehat{\mathcal{D}}_{k}$ by adding one new vertex.

Theorem 1.2. Let $S$ be a set of vertices in a graph $G \in \mathcal{D}_{k}$. Let $G^{\prime}$ be the graph obtained from $G$ by adding one new vertex adjacent to every vertex in $S$. Then $G^{\prime} \in \widehat{\mathcal{D}}_{k+1}$ if and only if $S$ is the set of vertices of degree $k+1$ in $G$.

Theorem 1.2 is proved in Section 6 along with various corollaries of Theorems 1.1 and 1.2 .

\footnotetext{
${ }^{1}$ All graphs considered in this paper are undirected, simple, and finite.

To contract an edge $v w$ in a graph $G$ means to delete $v w$, identify $v$ and $w$, and replace any parallel edges by a single edge. The contracted graph is denoted by $G / v w$. If $S \subseteq E(G)$ then $G / S$ is the graph obtained from $G$ by contracting each edge in $S$ (while edges in $S$ remain in $G$ ). The graph $G / S$ is called a contraction minor of $G$.

A graph $H$ is a minor of a graph $G$ if a graph isomorphic to $H$ can be obtained from a subgraph of $G$ by contracting edges. That is, $H$ can be obtained from $G$ by a sequence of edge contractions, edge deletions, or vertex deletions. For each vertex $v$ of $H$, the set of vertices of $G$ that are contracted into $v$ is called a branch set of $H$. A class $\mathcal{X}$ of graphs is minor-closed if every minor of every graph in $\mathcal{X}$ is also in $\mathcal{X}$, and some graph is not in $\mathcal{X}$.

The join of graphs $G$ and $H$, denoted by $G * H$, is the graph obtained by adding all possible edges between disjoint copies of $G$ and $H$. Let $\bar{G}$ denote the complement of a graph $G$.
} 
It is natural to expect that graphs in $\widehat{\mathcal{D}}_{k}$ are, in some sense, highly connected. For example for $k \leqslant 3$ all the graphs in $\widehat{\mathcal{D}}_{k}$ are $(k+1)$-connected. However, this is not true in general. In Section 2 we exhibit a graph in $\widehat{\mathcal{D}}_{4}$ with connectivity 1 . In fact, our third main result, proved in Section 7 , constructs graphs in $\widehat{\mathcal{D}}_{k}(k \geqslant 9)$ with arbitrary block structure.

Theorem 1.3. Let $T$ be the block decomposition tree of some graph. Then for some $k, T$ is the block decomposition tree of some graph in $\widehat{\mathcal{D}}_{k}$.

A complete characterisation of graphs in $\widehat{\mathcal{D}}_{k}$ is probably hopeless. So it is reasonable to restrict our attention to particular subsets of $\widehat{\mathcal{D}}_{k}$. A graph is complete c-partite if the vertices can be $c$-coloured so that two vertices are adjacent if and only if they have distinct colours. Let $K_{n_{1}, n_{2}, \ldots, n_{c}}$ be the complete $c$-partite graph with $n_{i}$ vertices in the $i$-th colour class. Since every graph in $\widehat{\mathcal{D}}_{k}$ for $k \leqslant 3$ is complete multipartite, it is natural to consider the complete multipartite graphs in $\widehat{\mathcal{D}}_{k}$. Our fourth main result characterises the complete multipartite graphs in $\widehat{\mathcal{D}}_{k}$.

Theorem 1.4. For all $k \geqslant 1$, a complete multipartite graph $G$ is in $\widehat{\mathcal{D}}_{k}$ if and only if for some $b \geqslant a \geqslant 1$ and $p \geqslant 2$,

$$
G=K_{a, \underbrace{b, \ldots, b}_{p},}^{,}
$$

such that $k+1=a+(p-1) b$ and if $p=2$ then $a=b$.

Theorem 1.4 is proved in Section 8. Moreover, we prove that the same characterisation holds for the minimal forbidden complete multipartite minors for the class of graphs for which every minor has connectivity at most $k$. And Theorem 8.9 is an analogous result for graphs of treewidth at most $k$ and pathwidth at most $k$.

Finally, note that our results can be interpreted in terms of the contraction degeneracy of a graph $G$, which is defined to be the maximum, taken over all minors $H$ of $G$, of the minimum degree of $H$. Thus, $G \in \widehat{\mathcal{D}}_{k}$ if and only if the contraction degeneracy of $G$ is at most $k$. See [2, 3, 18, 31, 32] for results about the computational complexity of determining the contraction degeneracy, and its relation to lower bounds on treewidth.

\section{Basics and Small Values of $k$}

This section gives some basic results about $\widehat{\mathcal{D}}_{k}$ and reviews what is known about $\widehat{\mathcal{D}}_{k}$ for small values of $k$. We have the following characterisation of graphs in $\widehat{\mathcal{D}}_{k}$.

Lemma 2.1. $G \in \widehat{\mathcal{D}}_{k}$ if and only if

(D1) $\delta(G)=k+1$,

(D2) every proper contraction minor of $G$ has minimum degree at most $k$,

(D3) $G$ is connected, and 
(D4) no two vertices both with degree at least $k+2$ are adjacent in $G$.

Proof. $(\Longrightarrow)$ Suppose that $G \in \widehat{\mathcal{D}}_{k}$. That is, $\delta(G) \geqslant k+1$ and every minor of $G$ has minimum degree at most $k$. In particular, every contraction minor of $G$ has minimum degree at most $k$, thus proving (D2). If $G$ is not connected then each component of $G$ is a proper minor with minimum degree $k+1$. This contradiction proves (D3). If adjacent vertices $v$ and $w$ both have degree at least $k+2$, then $G-v w$ is a proper minor of $G$ with minimum degree at least $k+1$. This contradiction proves (D4). In particular, some vertex has degree $k+1$. Thus $\delta(G)=k+1$ and (D1) holds.

$(\Longleftarrow)$ Suppose that conditions (D1)-(D4) hold. Suppose on the contrary that some proper minor of $G$ has minimum degree at least $k+1$. Let $H$ be such a minor with the maximum number of edges. Since $G$ is connected, $H$ can be obtained by edge contractions and edge deletions only. (Deleting a non-isolated vertex $v$ can be simulated by contracting one edge and deleting the other edges incident to $v$.) Condition (D4) implies that every edge has an endpoint with degree $k+1$, implying that every proper subgraph of $G$ has minimum degree at most $k$. Hence at least one edge of $G$ was contracted in the construction of $H$. Since $H$ was chosen with the maximum number of edges, no edges were deleted in the construction of $H$. That is, $H$ is a contraction minor. Condition (D2) implies that $H$ has minimum degree at most $k$. This contradiction proves that every proper minor of $G$ has minimum degree at most $k$. Thus condition (D1) implies that $G \in \widehat{\mathcal{D}}_{k}$.

Observe that Lemma 2.1 immediately implies that for all $k \geqslant 0$,

$$
K_{k+2} \in \widehat{\mathcal{D}}_{k}
$$

Now consider small values of $k$. Observe that $\mathcal{D}_{0}$ is the class of edgeless graphs, and $\widehat{\mathcal{D}}_{0}=\left\{K_{2}\right\}$. Similarly $\mathcal{D}_{1}$ is the class of forests, and $\widehat{\mathcal{D}}_{1}=\left\{K_{3}\right\}$. Graphs in $\mathcal{D}_{2}$ are often called series-parallel. $\widehat{\mathcal{D}}_{2}$ and $\widehat{\mathcal{D}}_{3}$ are easily determined; see Figure 1 .
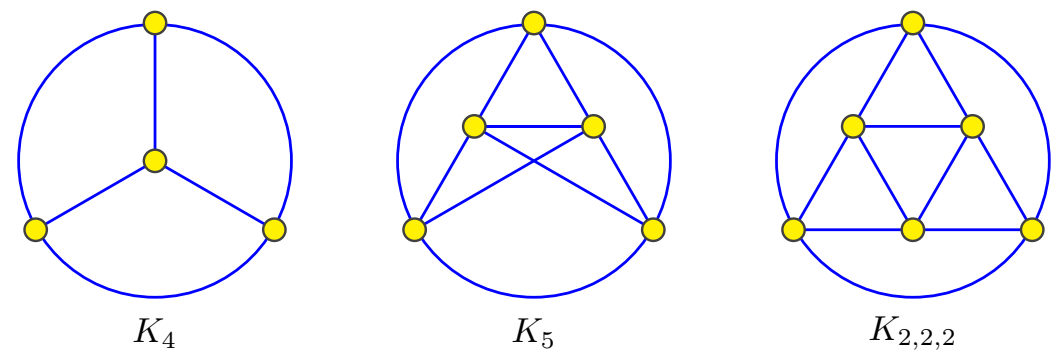

Figure 1: Graphs in $\widehat{\mathcal{D}}_{2}$ and $\widehat{\mathcal{D}}_{3}$.

Proposition 2.2. $\widehat{\mathcal{D}}_{2}=\left\{K_{4}\right\}$. 
Proof. By (1), $K_{4} \in \widehat{\mathcal{D}}_{2}$. Consider $G \in \widehat{\mathcal{D}}_{2}$. By Lemma 2.1, $G$ has minimum degree 3 . Dirac [9] proved that every graph with minimum degree at least 3 contains a $K_{4}$-minor; also see [16, 27, 33, 34]. Thus $G$ contains a $K_{4}$-minor. If $G \neq K_{4}$, then the $K_{4}$-minor in $G$ is not proper, implying $G \notin \widehat{\mathcal{D}}_{2}$ by Lemma 2.1. Hence $G \cong K_{4}$.

Proposition 2.3. $\widehat{\mathcal{D}}_{3}=\left\{K_{5}, K_{2,2,2}\right\}$.

Proof. By (1), $K_{5} \in \widehat{\mathcal{D}}_{3}$. Since $K_{2,2,2}$ is planar, every proper minor of $K_{2,2,2}$ is a planar graph on at most five vertices, which by Euler's Formula, has a vertex of degree at most 3. Thus $K_{2,2,2} \in \widehat{\mathcal{D}}_{3}$.

Consider $G \in \widehat{\mathcal{D}}_{3}$. By Lemma 2.1, $G$ has minimum degree 4. In Appendix A we prove that every graph with minimum degree at least 4 contains a 4 -connected minor ${ }^{2}$, Halin and Jung [17] proved that every 4-connected graph contains $K_{5}$ or $K_{2,2,2}$ as a minor. Thus $G$ contains $K_{5}$ or $K_{2,2,2}$ as a minor. Suppose on the contrary that $G$ is isomorphic to neither $K_{5}$ nor $K_{2,2,2}$. Then $G$ contains $K_{5}$ or $K_{2,2,2}$ as a proper minor. Thus $G$ contains a proper minor with minimum degree 4 , implying $G \notin \widehat{\mathcal{D}}_{4}$ by Lemma 2.1. Hence $G$ is isomorphic to $K_{5}$ or $K_{2,2,2}$.

Determining $\widehat{\mathcal{D}}_{4}$ is an open problem. But we do know nine graphs in $\widehat{\mathcal{D}}_{4}$, as illustrated in Figure 2, One of these graphs is the icosahedron I, which is the unique 5-regular planar triangulation (on twelve vertices). Mader [21] proved that every planar graph with minimum degree 5 contains $I$ as a minor. More generally, Mader [21] proved that every graph with minimum degree at least 5 contains a minor in $\left\{K_{6}, I, C_{5} * \overline{K_{3}}, K_{2,2,2,1}-e\right\}$, where $e$ is an edge incident to the degree-6 vertex in $K_{2,2,2,1}$. However, since $K_{2,2,2,1}-e$ has a degree- 4 vertex, it is not in $\widehat{\mathcal{D}}_{4}$. Fijavž [1] proved that every graph on at most 9 vertices with minimum degree at least 5 contracts to $K_{6}, K_{2,2,2,1}$ or $C_{5} * \overline{K_{3}}$. The graphs $G_{1}$ and $G_{2}$ are discussed further in Section 3 . The graphs $D_{1}$ and $D_{3}$ are due to Fijavž [11], while $D_{2}$ is due to Mader [21]. Note that $D_{1}, D_{2}$ and $D_{3}$ are not 5-connected. In fact, $D_{3}$ has a cut-vertex. It is an example of a more general construction given in Section 7 . In the language used there, $D_{3}$ is obtained from two copies of the single-horned graph $G_{5,4}$ by identifying the two horns.

Proposition 2.4. $\left\{K_{6}, I, C_{5} * \overline{K_{3}}, K_{1,2,2,2}, G_{1}, G_{2}, D_{1}, D_{2}, D_{3}\right\} \subseteq \widehat{\mathcal{D}}_{4}$.

Proof. This result was verified by computer. (The code is available from the authors upon request.) We now give manual proofs for some of these graphs.

$K_{6} \in \widehat{\mathcal{D}}_{4}$ by $(1)$.

$I$ is not in $\mathcal{D}_{4}$ since it is 5-regular. Every proper minor of $I$ is planar with at most eleven vertices. By Euler's Formula, every such graph has minimum degree at most 4, and is thus in $\mathcal{D}_{4}$. Hence $I \in \widehat{\mathcal{D}}_{4}$.

\footnotetext{
${ }^{2}$ This result was attributed by Maharry 23. to Halin and Jung [17. While the authors acknowledge their less than perfect understanding of German, Halin and Jung actually proved that every 4-connected graph contains $K_{5}$ or $K_{2,2,2}$ as a minor. This is confirmed by Tutte's review of the Halin and Jung paper in MathSciNet.
} 


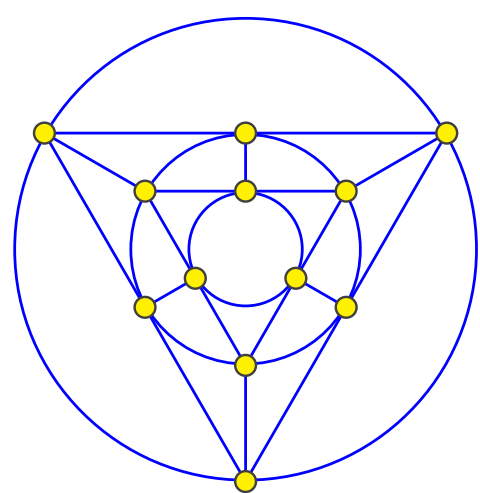

$I$

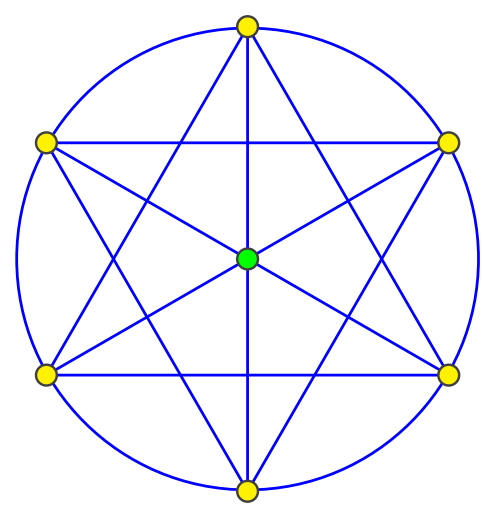

$K_{2,2,2,1}$

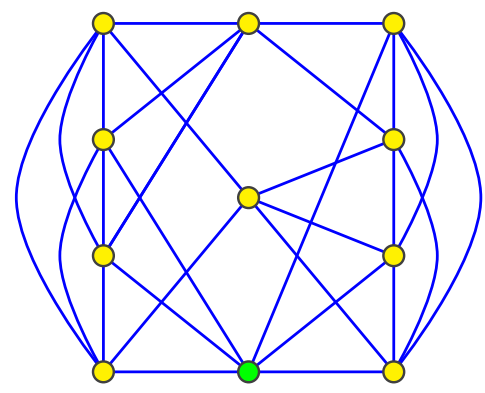

$D_{1}$

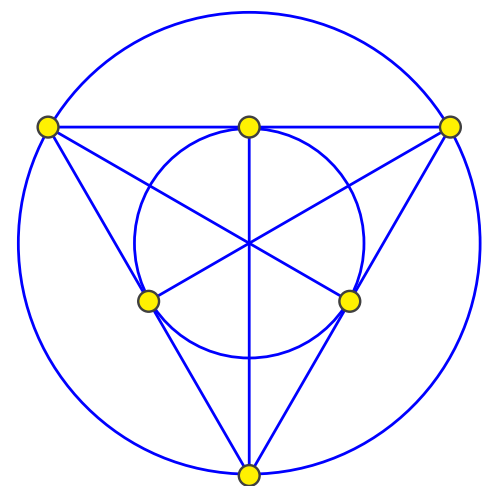

$K_{6}$

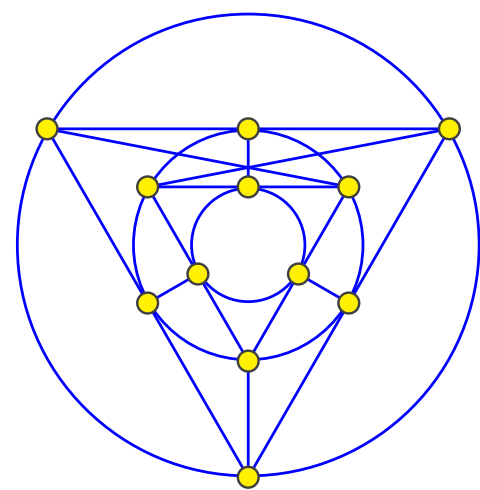

$G_{1}$

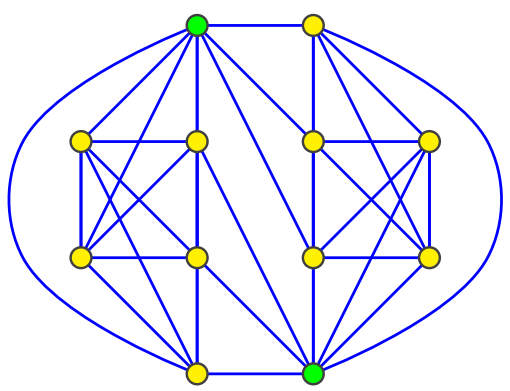

$D_{2}$

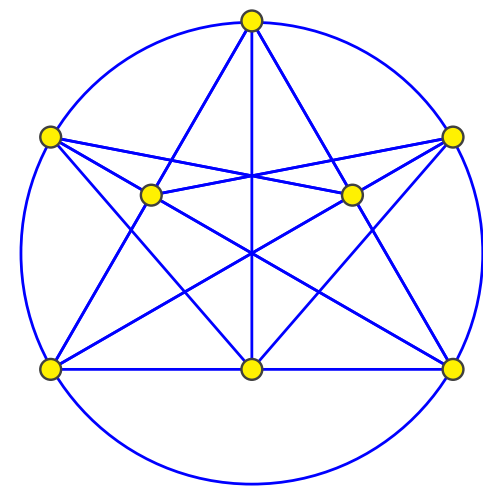

$C_{5} * \overline{K_{3}}$

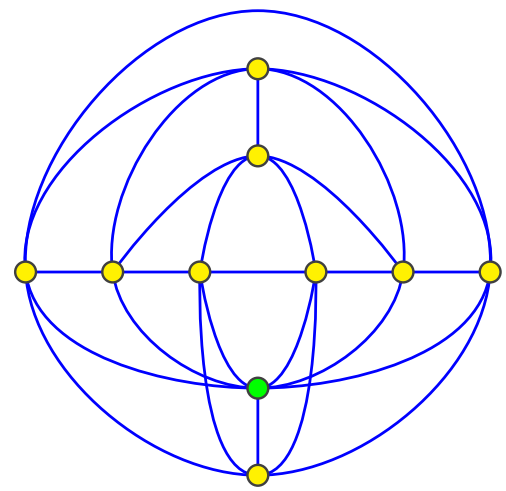

$G_{2}$

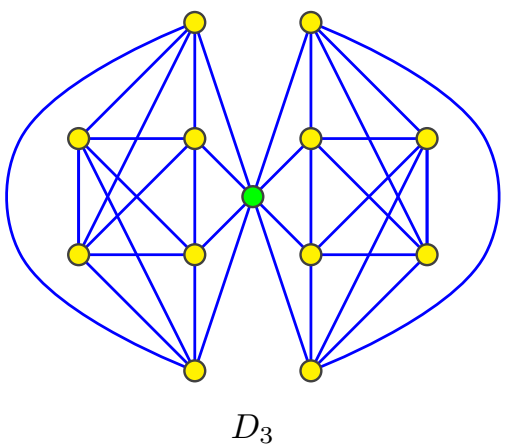

Figure 2: The known graphs in $\widehat{\mathcal{D}}_{4}$; vertices with degree more than 5 are highlighted.

We now prove that $C_{5} * \overline{K_{3}} \in \mathcal{D}_{4}$. Since $C_{5} * \overline{K_{3}}$ is 5-regular, conditions (D1), (D3) and (D4) hold in Lemma 2.1. Suppose that $C_{5} * \overline{K_{3}}$ contains a proper contraction minor $H$ with $\delta(H) \geqslant 5$. Thus $|V(H)| \geqslant 6$, and $H$ was obtained by at most two edge contractions. Since every edge of $C_{5} * \overline{K_{3}}$ is in a triangle with a degree-5 vertex, $H$ was obtained by exactly two edge contractions. Since each edge in the $C_{5}$ part of $C_{5} * \overline{K_{3}}$ is in three triangles, no edge in the $C_{5}$ part was contracted. Thus one contracted edge was $v w$ where 
$v \in C_{5}$ and $w \in \overline{K_{3}}$. Observe that $v w$ is in two triangles $v w x$ and $v w y$, where $x$ and $y$ are the neighbours of $v$ in $C_{5}$. Since both $x$ and $y$ have degree 4 in $G / v w$, some edge incident to $x$ and some edge incident to $y$ is contracted in $H$. This is impossible since $x$ and $y$ are not adjacent, and only one contraction besides $v w$ is allowed. This contradiction proves that every proper contraction minor of $G$ has minimum degree at most 4 . Thus condition (D2) holds for $C_{5} * \overline{K_{3}}$, and $C_{5} * \overline{K_{3}} \in \widehat{\mathcal{D}}_{4}$.

That $K_{1,2,2,2}$ is in $\widehat{\mathcal{D}}_{4}$ follows from Theorem 8.4 with $a=1$ and $b=2$ and $p=3$.

We now prove that $D_{3} \in \mathcal{D}_{4}$. Observe that conditions (D1), (D3) and (D4) in Lemma 2.1 hold for $D_{3}$. Suppose that $D_{3}$ contains a proper contraction minor $H$ with $\delta(H) \geqslant 5$. Thus $H=D_{3} / S$ for some $S \subseteq E(G)$ such that $|V(H)|=13-|S|$. Let $v$ be the cut-vertex in $D_{3}$. Let $G_{1}$ and $G_{2}$ be the subgraphs of $D_{3}$ such that $D_{3}=G_{1} \cup G_{2}$ where $V\left(G_{1}\right) \cap V\left(G_{2}\right)=\{v\}$. Let $S_{i}:=S \cap E\left(G_{i}\right)$. We have $\left|S_{i}\right| \leqslant\left|V\left(G_{i}\right)\right|-1=6$. Every edge of $D_{3}$ is in a triangle with a vertex distinct from $v$. Thus, if $\left|S_{i}\right|=1$ then some vertex in $H$ has degree at most 4 , which is a contradiction. If $2 \leqslant\left|S_{i}\right| \leqslant 5$ then $G_{i} / S$ has at least two and at most five vertices, and every vertex in $G_{i} / S$ (except possibly $v$ ) has degree at most 4, which is a contradiction. Thus $\left|S_{i}\right| \in\{0,6\}$. Now $\left|S_{1}\right|+\left|S_{2}\right|=|S| \leqslant 7$, as otherwise $H$ has at most five vertices. Thus $\left|S_{1}\right|=0$ and $\left|S_{2}\right|=6$ without loss of generality. Hence $H \cong G_{1}$, in which $v$ has degree 4 , which is a contradiction. Thus condition (D2) holds for $D_{3}$. Hence $D_{3} \in \widehat{\mathcal{D}}_{4}$.

\section{A General Setting}

The following general approach for studying minor-closed class was introduced by Fijavž [11. A graph parameter is a function $f$ that assigns a non-negative integer $f(G)$ to every graph $G$, such that for every integer $k$ there is some graph $G$ for which $f(G) \geqslant k$. Examples of graph parameters include minimum degree $\delta$, maximum degree $\Delta$, (vertex-) connectivity $\kappa$, edge-connectivity $\lambda$, chromatic number $\chi$, clique number $\omega$, independence number $\alpha$, treewidth tw, and pathwidth pw; see [8] for definitions.

For a graph parameter $f$ and a graph $G$, let $\widehat{f}(G)$ be the maximum of $f(H)$ taken over all minors $H$ of $G$. Then $\widehat{f}$ also is a graph parameter ${ }^{3}$. For example, $\widehat{\omega}(G)$ is the order of the largest clique minor in $G$, often called the Hadwiger number of $G$. Let

$$
\mathcal{X}_{f, k}:=\{G: \widehat{f}(G) \leqslant k\} .
$$

That is, $\mathcal{X}_{f, k}$ is the class of graphs $G$ such that $f(H) \leqslant k$ for every minor $H$ of $G$. Then $\mathcal{X}_{f, k}$ is minor-closed, and the set $\widehat{\mathcal{X}}_{f, k}$ of minimal forbidden minors is finite.

We have the following characterisation of graphs in $\widehat{\mathcal{X}}_{f, k}$, analogous to Lemma 2.1

Lemma 3.1. $G \in \widehat{\mathcal{X}}_{f, k}$ if and only if $f(G) \geqslant k+1$ and every proper minor $H$ of $G$ has $f(H) \leqslant k$.

\footnotetext{
${ }^{3}$ Let $\bar{f}(G)$ be the minimum of $f(H)$ where $G$ is a minor of $H$. Then the class of graphs $G$ with $\bar{f}(G) \leqslant k$ is minor-closed, and we can ask the same questions for $\bar{f}$ as for $\widehat{f}$. For example, the minor crossing number [4, 5, 6] fits into this framework.
} 
Proof. By definition, $G \in \widehat{\mathcal{X}}_{f, k}$ if and only if $G \notin \mathcal{X}_{f, k}$ but every proper minor of $G$ is in $\mathcal{X}_{f, k}$. That is, there exists a minor $H$ of $G$ with $f(H) \geqslant k+1$, but every proper minor $H$ of $G$ has $f(H) \leqslant k$. Thus the only minor $H$ of $G$ with $f(H) \geqslant k+1$ is $G$ itself.

Lemma 3.2. Let $\alpha$ and $\beta$ be graph parameters such that $\alpha(G) \leqslant \beta(G)$ for every graph G. Then $\mathcal{X}_{\beta, k} \subseteq \mathcal{X}_{\alpha, k}$ and $\left\{G: G \in \widehat{\mathcal{X}}_{\beta, k}, \alpha(G) \geqslant k+1\right\} \subseteq \widehat{\mathcal{X}}_{\alpha, k}$.

Proof. For the first claim, let $G$ be a graph in $\mathcal{X}_{\beta, k}$. Then $\beta(H) \leqslant k$ for every minor $H$ of $G$. By assumption, $\alpha(H) \leqslant \beta(H) \leqslant k$. Hence $G \in \mathcal{X}_{\alpha, k}$, implying $\mathcal{X}_{\beta, k} \subseteq \mathcal{X}_{\alpha, k}$.

For the second claim, suppose that $G \in \widehat{\mathcal{X}}_{\beta, k}$ and $\alpha(G) \geqslant k+1$. By Lemma 3.1 applied to $\beta, \beta(G) \geqslant k+1$ and every proper minor $H$ of $G$ has $\beta(H) \leqslant k$. By assumption, $\alpha(H) \leqslant \beta(H) \leqslant k$. Since $\alpha(G) \geqslant k+1$, Lemma 3.1 applied to $\alpha$ implies that $G \in \widehat{\mathcal{X}}_{\alpha, k}$.

Recall that $\delta$ and $\kappa$ are the graph parameters minimum degree and connectivity. Observe that $\mathcal{D}_{k}=\mathcal{X}_{\delta, k}$. Let

$$
\mathcal{C}_{k}:=\mathcal{X}_{\kappa, k}
$$

be the class of graphs for which every minor has connectivity at most $k$. For $k \leqslant 3$, we have $\mathcal{C}_{k}=\mathcal{D}_{k}$ and $\widehat{\mathcal{C}}_{k}=\widehat{\mathcal{D}}_{k}$. That is, $\widehat{\mathcal{C}_{1}}=\left\{K_{3}\right\}, \widehat{\mathcal{C}}_{2}=\left\{K_{4}\right\}$, and $\widehat{\mathcal{C}_{3}}=\left\{K_{5}, K_{2,2,2}\right\}$. Determining $\widehat{\mathcal{C}}_{4}$ is an open problem; Fijavž [1] conjectured that $\widehat{\mathcal{C}}_{4}=\left\{K_{6}, I, C_{5} *\right.$ $\left.\overline{K_{3}}, K_{1,2,2,2}, G_{1}, G_{2}\right\}$.

Dirac [10] proved that every 5-connected planar graph contains the icosahedron as a minor (which, as mentioned earlier, was generalised by Mader [21] for planar graphs of minimum degree 5). Thus the icosahedron is the only planar graph in $\widehat{\mathcal{C}}_{4}$. Fijavž [12] determined the projective-planar graphs in $\widehat{\mathcal{C}}_{4}$ to be $\left\{K_{6}, I, G_{1}, G_{2}\right\}$. Fijavž [14] determined the toroidal graphs in $\widehat{\mathcal{C}}_{5}$ to be $\left\{K_{7}, K_{2,2,2,2}, K_{3,3,3}, K_{9}-C_{9}\right\}$. See [13, 15] for related results. Also relevant is the large body of literature on contractibility; see the surveys [19, 22].

Let

$$
\mathcal{T}_{k}:=\{G: \operatorname{tw}(G) \leqslant k\} \quad \text { and } \mathcal{P}_{k}:=\{G: \operatorname{pw}(G) \leqslant k\}
$$

respectively be the classes of graphs with treewidth and pathwidth at most $k$. Since treewidth and pathwidth are minor-closed, $\mathcal{T}_{k}=\mathcal{X}_{\mathrm{tw}, k}$ and $\mathcal{P}_{k}=\mathcal{X}_{\mathrm{pw}, k}$. We have

$$
\kappa(G) \leqslant \delta(G) \leqslant \operatorname{tw}(G) \leqslant \operatorname{pw}(G)
$$

for every graph $G$; see [1, 8]. Thus Lemma 3.2 implies that

$$
\mathcal{P}_{k} \subseteq \mathcal{T}_{k} \subseteq \mathcal{D}_{k} \subseteq \mathcal{C}_{k}
$$

and

$$
\begin{aligned}
& \left\{G: G \in \widehat{\mathcal{D}}_{k}, \kappa(G) \geqslant k+1\right\} \subseteq \widehat{\mathcal{C}}_{k} \\
& \left\{G: G \in \widehat{\mathcal{T}}_{k}, \delta(G) \geqslant k+1\right\} \subseteq \widehat{\mathcal{D}}_{k} \\
& \left\{G: G \in \widehat{\mathcal{P}}_{k}, \operatorname{tw}(G) \geqslant k+1\right\} \subseteq \widehat{\mathcal{T}}_{k} .
\end{aligned}
$$

Thus the $(k+1)$-connected graphs that we show are in $\widehat{\mathcal{D}}_{k}$ are also in $\widehat{\mathcal{C}_{k}}$. In particular, Theorem 1.1 implies: 
Theorem 3.3. Every $(k+1)$-connected $(k+1)$-regular graph with less than $\frac{4}{3}(k+2)$ vertices is in $\widehat{\mathcal{C}_{k}}$.

The relationship between $\widehat{\mathcal{C}_{k}}$ and $\widehat{\mathcal{D}}_{k}$ is an interesting open problem.

Open Problem 3.4. Is $\widehat{\mathcal{C}_{k}} \subseteq \widehat{\mathcal{D}}_{k}$ for all $k$ ? Is $\widehat{\mathcal{C}_{k}}=\left\{G: G \in \widehat{\mathcal{D}}_{k}, \kappa(G)=k+1\right\}$ for all $k$ ?

Note that $\widehat{\mathcal{D}}_{4} \neq \widehat{\mathcal{C}}_{4}$ since there are graphs in $\widehat{\mathcal{D}}_{4}$ with connectivity 1 ; see Section 7 .

\section{General Values of $k$}

Let $G$ be a graph. A vertex of $G$ is low-degree if its degree equals the minimum degree of $G$. A vertex of $G$ is high-degree if its degree is greater than the minimum degree of $G$. Recall that every graph in $\widehat{\mathcal{D}}_{k}$ has minimum degree $k+1$. Thus a vertex of degree $k+1$ in a graph in $\widehat{\mathcal{D}}_{k}$ is low-degree; every other vertex is high-degree. Lemma 2.1 implies that for every graph $G \in \widehat{\mathcal{D}}_{k}$, the high-degree vertices in $G$ form an independent set.

Proposition 4.1. Every graph $G \in \widehat{\mathcal{D}}_{k}$ has at least $k+2$ low-degree vertices (of degree $k+1)$.

Proof. Suppose on the contrary that $G$ has at most $k+1$ low-degree vertices. By Lemma 2.1, each high-degree vertex is only adjacent to low-degree vertices. Since a highdegree vertex has degree at least $k+2$, there are no high-degree vertices. Thus $G$ has at most $k+1$ vertices. Thus $G$ has maximum degree at most $k$, which is a contradiction.

For a set $S$ of vertices in a graph $G$, a common neighbour of $S$ is a vertex in $V(G)-S$ that is adjacent to at least two vertices in $S$. A common neighbour of an edge $v w$ is a common neighbour of $\{v, w\}$. Common neighbours are important because of the following observation.

Observation 4.2. Let $v w$ be an edge of a graph $G$ with $p$ common neighbours. Let $H$ be the graph obtained from $G$ by contracting vw into a new vertex $x$. Then

$$
\operatorname{deg}_{H}(x)=\operatorname{deg}_{G}(v)+\operatorname{deg}_{G}(w)-p-2 .
$$

For every common neighbour y of vw,

$$
\operatorname{deg}_{H}(y)=\operatorname{deg}_{G}(y)-1 .
$$

For every other vertex $z$ of $H$,

$$
\operatorname{deg}_{H}(z)=\operatorname{deg}_{G}(z) .
$$

Proposition 4.3. For every graph $G \in \widehat{\mathcal{D}}_{k}$, every edge vw of $G$ has a low-degree common neighbour. 
Proof. If $k=1$ then $G=K_{3}$ and the result is trivial. Now assume that $k \geqslant 2$.

Suppose on the contrary that for some edge $v w$ of $G$, every common neighbour of $v w$ (if any) is high-degree. By Lemma 2.1, at least one of $v$ and $w$ is low-degree (with degree $k+1)$. Thus $v$ and $w$ have at most $k$ common neighbours. Let $u_{1}, \ldots, u_{p}$ be the common neighbours of $v$ and $w$, where $0 \leqslant p \leqslant k$.

Let $H$ be the graph obtained from $G$ by contracting $v w$ into a new vertex $x$. The degree of each vertex of $G$ is unchanged in $H$, except for $v, w$, and each $u_{i}$. Since $\operatorname{deg}_{G}\left(u_{i}\right) \geqslant k+2$, we have $\operatorname{deg}_{H}\left(u_{i}\right) \geqslant k+1$. By Observation 4.2 ,

$$
\operatorname{deg}_{H}(x)=\operatorname{deg}_{G}(v)+\operatorname{deg}_{G}(w)-p-2 \geqslant 2(k+1)-p-2=2 k-p .
$$

Thus if $p \leqslant k-1$ then $\operatorname{deg}_{H}(x) \geqslant k+1$ and $H$ is a proper minor of $G$ with minimum degree at least $k+1$, implying $G \notin \widehat{\mathcal{D}}_{k}$.

Otherwise $p=k$, implying both $v$ and $w$ are low-degree vertices whose only neighbours are each other and the high-degree vertices $u_{1}, \ldots, u_{k}$. Let $J$ be the graph obtained from $G$ by contracting $v, w, u_{1}$ into a new vertex $y$. Since each neighbour of $v$ is high-degree and each neighbour of $w$ is high-degree, if a vertex (other than $v, w, u_{1}$ ) is adjacent to at least two of $v, w, u_{1}$ then it is high-degree. Since no two high-degree vertices are adjacent, the only vertices (other than $v, w, u_{1}$ ) that are adjacent to at least two of $v, w, u_{1}$ are $u_{2}, \ldots, u_{k}$. Thus every vertex in $J$ (possibly except $y$ ) has degree at least $k+1$. Now $u_{1}$ has at least $k$ neighbours in $G$ outside of $\left\{v, w, u_{2}, \ldots, u_{k}\right\}$. Thus $\operatorname{deg}_{J}(y) \geqslant k+(k-1) \geqslant k+1$, and $J$ is a proper minor of $G$ with minimum degree at least $k+1$, implying $G \notin \widehat{\mathcal{D}}_{k}$.

The next result says that for graphs in $\widehat{\mathcal{D}}_{k}$, every sufficiently sparse connected induced subgraph has a common neighbour.

Proposition 4.4. For every graph $G \in \widehat{\mathcal{D}}_{k}$, for every connected induced subgraph $H$ of $G$ with $n$ vertices and $m \leqslant \frac{1}{2}(k+1)(n-1)$ edges, there exists a vertex $x$ in $G-H$ adjacent to at least $\operatorname{deg}_{G}(x)-k+1 \geqslant 2$ vertices in $H$.

Proof. Suppose that for some connected induced subgraph $H$ with $n$ vertices and $m \leqslant$ $\frac{1}{2}(k+1)(n-1)$ edges, every vertex $x$ in $G-H$ is adjacent to at most $\operatorname{deg}_{G}(x)-k$ vertices in $H$. Let $G^{\prime}$ be the graph obtained from $G$ by contracting $H$ into a single vertex $v$. The degree of every vertex in $G-H$ is at least $\operatorname{deg}_{G}(x)-\left(\operatorname{deg}_{G}(x)-k\right)+1=k+1$ in $G^{\prime}$. Since $G$ has minimum degree $k+1$, we have

$$
\operatorname{deg}_{G^{\prime}}(v)=\left(\sum_{w \in V(H)} \operatorname{deg}_{G}(w)\right)-2 m \geqslant n(k+1)-2 m \geqslant k+1 .
$$

Thus $G^{\prime}$ is a proper minor of $G$ with minimum degree at least $k+1$. Hence $G \notin \widehat{\mathcal{D}}_{k}$. This contradiction proves the result.

Corollary 4.5. For every graph $G \in \widehat{\mathcal{D}}_{k}$, for every clique $C$ of $G$ with at most $k+1$ vertices, there exists a vertex in $V(G)-C$ adjacent to at least two vertices of $C$. 


\section{Small Regular Graphs are in $\widehat{\mathcal{D}}_{k}$}

In this section we show that that every $(k+1)$-regular graph with sufficiently few vertices is in $\widehat{\mathcal{D}}_{k}$. Moreover, the bound on the number of vertices is tight.

Lemma 5.1. Let $G$ be a connected $(k+1)$-regular graph on $n$ vertices. If every edge of $G$ is in at least $2 n-2 k-5$ triangles, then $G \in \widehat{\mathcal{D}}_{k}$.

Proof. By assumption, conditions (D1), (D3) and (D4) of Lemma 2.1 are satisfied by $G$. Suppose on the contrary that $H$ is a contraction minor of $G$ with minimum degree at least $k+1$. Let $S$ be the set of vertices of $G$ that are incident to an edge contracted in the construction of $H$. Let $v w$ be one such edge. We have $|S| \leqslant 2(n-|V(H)|) \leqslant 2 n-2 k-4$. By assumption, there is a set $T$ of vertices of $G$ that are adjacent to both $v$ and $w$, and $|T| \geqslant 2 n-2 k-5 \geqslant|S|-1$. Thus there is at least one vertex $x \in T-(S-\{v, w\})$, which is a vertex of $H$. Since $x$ is adjacent to both endpoints of the contracted edge $v w$, $\operatorname{deg}_{H}(x) \leqslant k$. This contradiction proves condition (D2) for $G$. Lemma 2.1 implies that $G \in \widehat{\mathcal{D}}_{k}$.

Lemma 5.2. For every $(k+1)$-regular graph $G$ on $n$ vertices, every edge vw of $G$ is in at least $2 k+2-n$ triangles.

Proof. Say $v w$ is in $t$ triangles. Thus $v$ and $w$ have $t$ common neighbours. Thus $v$ has $k-t$ neighbours not adjacent to $w$, and $w$ has $k-t$ neighbours not adjacent to $v$. Thus $n \geqslant 2+t+2(k-t)=2 k+2-t$, implying $t \geqslant 2 k+2-n$.

Theorem 5.3. Every $(k+1)$-regular graph $G$ with $n<\frac{4}{3}(k+2)$ vertices is in $\widehat{\mathcal{D}}_{k}$.

Proof. Every disconnected $(k+1)$-regular graph has at least $2 k+4$ vertices. Since $n<$ $2 k+4$ we can assume that $G$ is connected. By Lemma 5.2, every edge of $G$ is in at least $2 k+2-n$ triangles. Now $2 k+2-n \geqslant 2 n-2 k-5$ since $n \leqslant \frac{1}{3}(4 k+7)$. Thus every edge of $G$ is in at least $2 n-2 k-5$ triangles. By Lemma 5.1. $G \in \widehat{\mathcal{D}}_{k}$.

Theorem 5.3 is best possible in the following sense.

Proposition 5.4. For all $k \equiv 1(\bmod 3)$ there is a $(k+1)$-regular graph $G$ on $n=\frac{4}{3}(k+2)$ vertices that is not in $\widehat{\mathcal{D}}_{k}$.

Proof. Let $p:=\frac{1}{3}(k+2)$. Then $p \in \mathbb{Z}$. Let $G$ be the graph whose complement $\bar{G}$ is the disjoint union of $K_{p, p}$ and $K_{p, p}$. Then $G$ has $4 p=n$ vertices, and every vertex has degree $n-1-p=k+1$. Observe that $G$ contains a matching $M$ of $p$ edges (between the two $K_{p, p}$ subgraphs in $\bar{G}$ ), such that every vertex is adjacent to at least one endpoint of every edge in $M$. Contracting each edge in $M$ we obtain a $K_{3 p}$-minor in $G$, which has minimum degree $k+1$. Thus $G \notin \widehat{\mathcal{D}}_{k}$.

Theorem 5.3 can be rewritten in terms of complements.

Corollary 5.5. If $G$ is an $r$-regular graph on $n \geqslant 4 r+1$ vertices, then $\bar{G} \in \widehat{\mathcal{D}}_{n-r-2}$. 


\section{A Construction}

We now describe how a graph in $\widehat{\mathcal{D}}_{k+1}$ can be constructed from a graph in $\widehat{\mathcal{D}}_{k}$. Let $G^{+}$ be the graph obtained from a graph $G$ by adding one new vertex that is adjacent to each vertex of minimum degree in $G$. If $G \in \widehat{\mathcal{D}}_{k}$ then the vertices of minimum degree are the low-degree vertices.

Lemma 6.1. If $G \in \widehat{\mathcal{D}}_{k}$ then $G^{+} \in \widehat{\mathcal{D}}_{k+1}$.

Proof. Let $v$ be the vertex of $G^{+}-G$. Every low-degree vertex in $G$ has degree $k+1$, and thus has degree $k+2$ in $G^{+}$. Every high-degree vertex in $G$ has degree at least $k+2$, which is unchanged in $G^{+}$. By Proposition 4.1. $G$ has at least $k+2$ low-degree vertices. Thus $v$ has degree at least $k+2$ in $G^{+}$. Thus $G^{+}$has minimum degree $k+2$. Suppose on the contrary that $G \in \widehat{\mathcal{D}}_{k}$ but $G^{+} \notin \widehat{\mathcal{D}}_{k+1}$. Thus there is a proper minor $H$ of $G^{+}$with minimum degree at least $k+2$. If $v$ is not in a branch set of $H$, then $H$ is a minor of $G$, implying $H$ has minimum degree at most $k+1$, which is a contradiction. Now assume that $v$ is in some branch set $B$ of $H$. (Think of $B$ simultaneously as a vertex of $H$ and as a set of vertices of $G^{+}$.) Now $H-B$ is a minor of $G$. If $H-B$ is $G$, then $B=\{v\}$ and $H$ is not a proper minor of $G^{+}$. Thus $H-B$ is a proper minor of $G$. Since $G \in \widehat{\mathcal{D}}_{k}, H-B$ has a vertex $X$ of degree at most $k$. Thus $X$ has degree at most $k+1$ in $H$, which is a contradiction.

We also have a converse result.

Lemma 6.2. Let $S$ be a set of vertices in a graph $G \in \mathcal{D}_{k}$. Let $G^{\prime}$ be the graph obtained from $G$ by adding one new vertex $v$ adjacent to every vertex in $S$. If $G^{\prime} \in \widehat{\mathcal{D}}_{k+1}$ then $S$ is the set of low-degree vertices in $G$.

Proof. Suppose that $G^{\prime} \in \widehat{\mathcal{D}}_{k+1}$. If some low-degree vertex $x$ of $G$ is not in $S$, then $\operatorname{deg}_{G^{\prime}}(x)=k+1$ and $G^{\prime} \notin \widehat{\mathcal{D}}_{k+1}$. Now assume that every low-degree vertex of $G$ is in $S$. Suppose on the contrary that some high-degree vertex $y$ of $G$ is in $S$. Thus $\operatorname{deg}_{G}(y) \geqslant k+2$, implying $\operatorname{deg}_{G^{\prime}}(y) \geqslant k+3$. By Proposition 4.1 there are at least $k+2$ low-degree vertices of $G$, all of which are adjacent to $v$ in $G^{\prime}$. Thus $\operatorname{deg}_{G^{\prime}}(v) \geqslant k+3$. Hence $v$ and $y$ are adjacent vertices of degree at least $k+3$ in $G^{\prime}$. Therefore $G^{\prime} \notin \widehat{\mathcal{D}}_{k+1}$ by Lemma 2.1. This contradiction proves that no high-degree vertex of $G$ is in $S$. Therefore $S$ is the set of low-degree degree vertices.

Observe that Lemmas 6.1 and 6.2 together prove Theorem 1.2 . Lemma 6.1 generalises as follows. For a non-negative integer $p$, let $G^{+p}$ be the graph obtained from a graph $G$ by adding $p$ independent vertices, each adjacent to every vertex in $G$.

Lemma 6.3. Let $G$ be a $(k+1)$-regular $n$-vertex graph in $\widehat{\mathcal{D}}_{k}$. Then $G^{+p} \in \widehat{\mathcal{D}}_{k+p}$ whenever $0 \leqslant p \leqslant n-k-1$.

Proof. Every vertex of $G$ has degree $k+1+i$ in $G^{+i}$. Every vertex of $G^{+i}-G$ has degree $n$ in $G^{+i}$. Thus, if $n>k+1+i$ then the vertices of minimum degree in $G^{+i}$ are exactly the vertices of $G$. Thus $G^{+i}=\left(G^{+(i-1)}\right)^{+}$whenever $1 \leqslant i \leqslant n-k-1$. By induction on $i$, applying Lemma 6.1 at each step, we conclude that $G^{+i} \in \widehat{\mathcal{D}}_{k+i}$ and $G^{+p} \in \widehat{\mathcal{D}}_{k+p}$. 
Theorem 5.3 and Lemma 6.3 imply:

Corollary 6.4. Let $G$ be a $(k+1)$-regular graph with $n<\frac{4}{3}(k+2)$ vertices. Then $G^{+p} \in \widehat{\mathcal{D}}_{k+p}$ whenever $0 \leqslant p \leqslant n-k-1$.

Corollary 6.4 implies:

Lemma 6.5. Let $L(G)$ denote the set of minimum degree vertices in a graph $G$. Let $p:=|G-L(G)|$. Suppose that

- the minimum degree of $G$ is $k+1$, and

- $|L(G)|<\frac{4}{3}(k+2-p)$, and

- $V(G)-L(G)$ is an independent set of $G$, and

- every vertex in $V(G)-L(G)$ dominates $L(G)$.

Then $G \in \widehat{\mathcal{D}}_{k}$.

Proof. Let $X$ be the subgraph of $G$ induced by the vertices of degree $k+1$. Thus $X$ is $(r+1)$-regular, where $r=k-p$. Say $X$ has $n$ vertices. By assumption, $n<\frac{4}{3}(k+2-p)=$ $\frac{4}{3}(r+2)$. The high-degree vertices of $G$ have degree $n$, and the low-degree vertices of $G$ have degree $r+1+p$. Thus $n>r+1+p$. That is, $p<n-r-1$. Thus, by Corollary 6.4. we have $G=X^{+p} \in \widehat{\mathcal{D}}_{r+p}=\widehat{\mathcal{D}}_{k}$.

\section{Block Structure}

In this section we show that graphs in $\widehat{\mathcal{D}}_{k}$ can have an arbitrary block decomposition tref 4 Theorems 7.7 and 7.8 are the main results. Note that every graph in $\widehat{\mathcal{D}}_{k}$ has no cut-edge (except $K_{2}$ ), since a cut-edge can be contracted without decreasing the minimum degree.

A low-high tree is a tree $T$ that admits a bipartition $V(T)=V_{\ell} \cup V_{h}$, such that every vertex in $V_{\ell}$ has degree at most 2 , and every vertex in $V_{h}$ has degree at least 2 . Vertices in $V_{\ell}$ are called low, and vertices in $V_{h}$ are called high. Since every leaf in a low-high tree is low, every low-high tree is a block decomposition tree.

In the following discussion, let $T$ be a low-high tree. Let $L$ be the set of leaves in $T$. Let $r$ be an arbitrary high vertex of $T$, called the root. For each edge $v w \in E(T)$, let $\operatorname{dist}(r, v w):=\min \{\operatorname{dist}(r, v), \operatorname{dist}(r, w)\}$. Let $B$ be the set of edges of $T$ at even distance

\footnotetext{
${ }^{4}$ Let $G$ be a connected graph. Let $B$ denote the set of blocks of $G$ (that is, cut-edges and maximal 2-connected components). Let $C$ denote the set of cut-vertices of $G$. The block decomposition tree of $G$ is the tree $T$ where $V(T)=B \cup C$, and $b c \in E(T)$ whenever the block $b$ contains $c$. A block decomposition tree is a tree that is isomorphic to a block decomposition tree of some graph. The bipartition of a tree $T$ is the partition of $V(T)$ obtained from a proper 2-colouring of $T$. Since every cut-vertex is contained in at least two blocks, every leaf of a block decomposition tree $T$ belongs to the same bipartition class of $T$. Conversely, if a tree $T$ admits a bipartition of its vertices such that all leaves lie in the same bipartition class, then $T$ is a block decomposition tree.
} 
from $r$. Call these edges blue. Similarly let $R:=E(T)-B$ be the set of red edges in $T$. Since $r$ is high and each leaf is low, each leaf is at odd distance from $r$. Thus each edge incident with a leaf is blue.

Lemma 7.1. The number of blue edges $|B|$ and the number of red edges $|R|$ do not depend on the choice of $r$.

Proof. It is enough to show that $|B|$ and $|R|$ do not change if we choose an alternative root $r^{\prime}$ at distance 2 from $r$. Let $R^{\prime}$ and $B^{\prime}$ be the sets of red and blue edges with respect to $r^{\prime}$. Let $x$ be the common neighbour of $r$ and $r^{\prime}$. Thus $r x \in B-B^{\prime}$ and $r^{\prime} x \in B^{\prime}-B$. Apart from these edges, $B$ and $B^{\prime}$ do not differ. Hence $|B|=\left|B^{\prime}\right|$, and also $|R|=\left|R^{\prime}\right|$.

Define

$$
d:=4|L|+2|R| .
$$

Since $T$ has at least two leaves, $d \geqslant 8$. By Lemma 7.1, $d$ does not depend on the choice of $r$.

For each edge $e=v w$ of $T$ such that $\operatorname{dist}(r, v)=\operatorname{dist}(r, w)-1$, let $T_{e}$ be the maximal rooted subtree of $T$ containing $v w$, and no other neighbour of $v$.

Define the function $\varphi: E(T) \rightarrow \mathbb{N}$ as follows. For each blue edge $e$ in $T$, define

$$
\varphi(e):=4\left|L \cap E\left(T_{e}\right)\right|+2\left|R \cap E\left(T_{e}\right)\right| .
$$

Now consider a red edge $v w$ in $T$ with $\operatorname{dist}(r, v)=\operatorname{dist}(r, w)-1$. Thus $\operatorname{dist}(r, v)$ is odd, $v$ is low, and $\operatorname{deg}(v)=2$. Let $u v$ be the blue edge incident to $v$. Define

$$
\varphi(v w):=d+2-\varphi(u v) .
$$

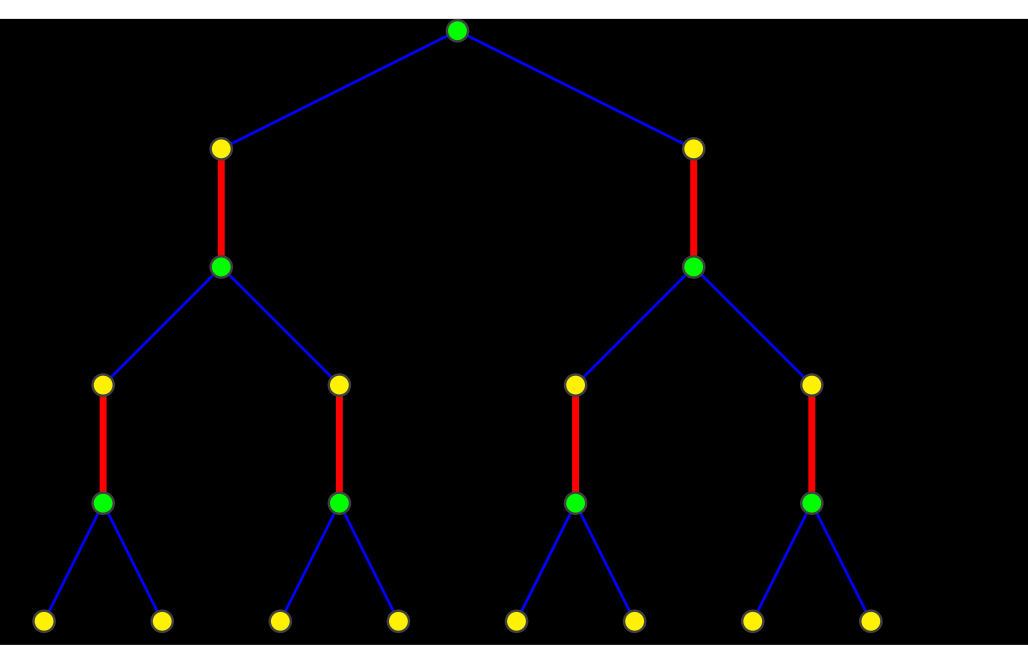

Figure 3: An example of the edge labelling $\varphi$ with $|R|=6$ and $|B|=14$ and $|L|=8$ and $d=2 \cdot 6+4 \cdot 8=44$. Red edges are drawn thick.

The next lemma immediately follows from (6). 
Lemma 7.2. If $v$ is a low vertex of degree 2 and $v$ is incident with edges $e$ and $f$, then $\varphi(e)+\varphi(f)=d+2$.

The sum of $\varphi$ values around a high vertex is also constant.

Lemma 7.3. Let $v$ be a high vertex and let $E_{v}$ be the set of edges incident with $v$. Then $\sum_{e \in E_{v}} \varphi(e)=d$.

Proof. First suppose that $v=r$. Then

$$
\sum_{r x \in E_{r}} \varphi(r x)=\sum_{r x \in E_{r}} 4\left|L \cap E\left(T_{r x}\right)\right|+2\left|R \cap E\left(T_{r x}\right)\right|=4|L \cap E(T)|+2|R \cap E(T)|=d .
$$

Now assume that $v \neq r$. Since $v$ is high, $\operatorname{dist}(v, r)$ is even, and $v$ is incident to one red edge $u v$ (where $u$ is the neighbour of $v$ closer to $r$ than $v$ ). Thus $u$ is low, and $\operatorname{deg}(u)=2$. Let $t$ be the other neighbour of $u$. Let $e_{1}, \ldots, e_{k}$ be the blue edges incident to $v$. Then

$$
\begin{aligned}
\sum_{e \in E_{v}} \varphi(e) & =\varphi(u v)+\sum_{i=1}^{k} \varphi\left(e_{i}\right) \\
& =d+2-\varphi(t u)+\sum_{i=1}^{k} \varphi\left(e_{i}\right) \\
& =d+2-4\left|L \cap E\left(T_{t u}\right)\right|-2\left|R \cap E\left(T_{t u}\right)\right|+\sum_{i=1}^{k} 4\left|L \cap E\left(T_{e_{i}}\right)\right|+2\left|R \cap E\left(T_{e_{i}}\right)\right|
\end{aligned}
$$

Observe that $L \cap E\left(T_{t u}\right)=\bigcup_{i} L \cap E\left(T_{e_{i}}\right)$ and $R \cap E\left(T_{t u}\right)-\bigcup_{i} R \cap E\left(T_{e_{i}}\right)=\{u v\}$. Thus

$$
\sum_{e \in E_{v}} \varphi(e)=d+2-2=d
$$

Observe that, in principle, the definition of $\varphi$ depends on the choice of $r$. However, this is not the case.

Lemma 7.4. Let $r$ and $r^{\prime}$ be high vertices of $T$, and let $\varphi$ and $\varphi^{\prime}$ be the functions defined above using $r$ and $r^{\prime}$ as roots, respectively. Then $\varphi=\varphi^{\prime}$.

Proof. Since $T$ is connected, it is enough to show that $\varphi=\varphi^{\prime}$ whenever $\operatorname{dist}\left(r, r^{\prime}\right)=2$. Let $x$ be the common neighbour of $r$ and $r^{\prime}$. Let $B^{\prime}$ be the set of blue edges with respect to $r^{\prime}$. Now $B$ and $B^{\prime}$ (as well as $R$ and $R^{\prime}$ ) differ only in $r x$ and $r^{\prime} x$. Since (5) only considers $\varphi$ and $\varphi^{\prime}$ values of blue edges away from the root, $\varphi(e)=\varphi^{\prime}(e)$ for each $e \in B \cap B^{\prime}$. Since each edge incident with $r$ or $r^{\prime}$ apart from $r x$ and $r^{\prime} x$ is in $B \cap B^{\prime}$, and since $d$ is invariant, (6) shows that $\varphi$ and $\varphi^{\prime}$ match on every edge in $R \cap R^{\prime}$. Finally Lemma 7.3 implies that $\varphi$ and $\varphi^{\prime}$ also match on edges between $r x$ and $r^{\prime} x$. 
Lemma 7.5. $\varphi(e) \geqslant 4$ for every edge $e \in E(T)$.

Proof. While the colour of an edge $e$ may depend on the choice of $r$, Lemma 7.4 says that $\varphi(e)$ does not depend on the choice of $r$. Every edge can be made blue for an appropriate choice of $r$, and $\varphi(e) \geqslant 4$ for every blue edge $e$ by (5).

And now for something completely different. Let $e=u_{1} u_{2}$ and $f=u_{3} u_{4}$ be two independent edges in the complete graph $K_{d+1}$, where $d \geqslant 4$. The single-horned graph $G_{d, 4}$ is obtained from $K_{d+1}$ by adding a new vertex $x$, connecting $x$ to $u_{1}, u_{2}, u_{3}$, and $u_{4}$ and removing edges $e$ and $f$. Observe that $\operatorname{deg}(x)=4$. Call $x$ the horn of $G_{d, 4}$. Call the remaining vertices the original vertices of $G_{d, 4}$, which all have degree $d$.

Let $a, b \geqslant 4$ be even integers such that $d=a+b-2$. Choose matchings $M_{a}$ and $M_{b}$ with $\frac{a}{2}$ and $\frac{b}{2}$ edges, respectively, that cover all the vertices of $K_{d+1}$. Hence $M_{a}$ and $M_{b}$ share exactly one vertex. Take two new vertices $x_{a}$ and $x_{b}$ and join $x_{a}$ to every vertex of $M_{a}$ and $x_{b}$ to every vertex of $M_{b}$. Next delete the edges of $M_{a}$ and $M_{b}$. The resulting graph is called the double-horned graph $G_{d, a, b}$. As above, $x_{a}$ and $x_{b}$ are called the horns of $G_{d, a, b}$, and the remaining vertices, all of degree $d$, are the original vertices.

Let $e=u v$ be an edge in a single- or double-horned graph $G$. If $u$ or $v$ is a horn in $G$, then the vertex $u v$ is a horn in $G / e$ and is original otherwise. Inductively, we can define horns and original vertices for every contraction minor of a horned graph.

Lemma 7.6. Let $G^{\prime}$ be a proper contraction minor of a horned graph $G_{d, 4}$ or $G_{d, a, b}$. If $G^{\prime}$ contains an original vertex, then some original vertex of $G^{\prime}$ has degree less than $d$.

Proof. We shall leave the proof for $G_{d, 4}$ to the keen reader. Let $G$ be the doubly horned graph $G_{d, a, b}$, and let $F \subseteq E(G)$ such that $G / F=G^{\prime}$. If $|F| \geqslant 3$, then $G^{\prime}$ has at most $d$ vertices, and all its vertices have degree less than $d$. Now assume that $|F| \leqslant 2$.

Let $e=u v$ be an edge connecting a pair of original vertices. There are at least $7 \leqslant a+b-1$ original vertices in $G$ and at least three original vertices are connected with both $u$ and $v$. Thus $G / e$ has at least three original vertices of degree less than $d$, which cannot all be eliminated by a single additional contraction. Hence every edge in $F$ is incident with a horn. Let $x$ be a horn incident with $e$. At least two neighbours of $x$ (which are original vertices) have degree less than $d$ in $G / e$, yet by the above argument, the edge between them cannot be contracted.

We are now ready to state the first theorem of this section.

Theorem 7.7. For every low-high tree $T$, there is an integer $d$ and a graph $G$ such that:

(G1) $G$ is d-regular,

(G2) $T$ is the block decomposition tree of $G$,

(G3) $8 \leqslant d \leqslant 4|E(T)|$, and

(G4) $G \in \widehat{\mathcal{D}}_{d+1}$. 
Proof. Adopt the above notation. Let $d:=4|L|+2|R|$. By construction, $d \geqslant 8$. Note that $d \leqslant 4|E(T)|$ with equality only if $T$ is a star.

For every leaf $u$ of $T$, let $G_{u}$ be a copy of the single-horned graph $G_{d, 4}$. For every nonleaf low vertex $v$ of $T$ incident with edges $e$ and $f$, let $G_{v}$ be a copy of the double-horned graph $G_{d, a, b}$, where $a:=\varphi(e)$ and $b:=\varphi(f)$. Note that $a, b \geqslant 4$ by Lemma 7.5.

Observe that there is a natural correspondence between the set of horns in the above graphs and their degrees, and between $E(T)$ and their $\varphi$ values. As illustrated in Figure 4 , identifying horns wherever the edges in $T$ have a common (high) end-vertex gives rise to a $d$-regular graph $G$ (by Lemma 7.3). Hence $G$ satisfies (G1), (G2) and (G3).

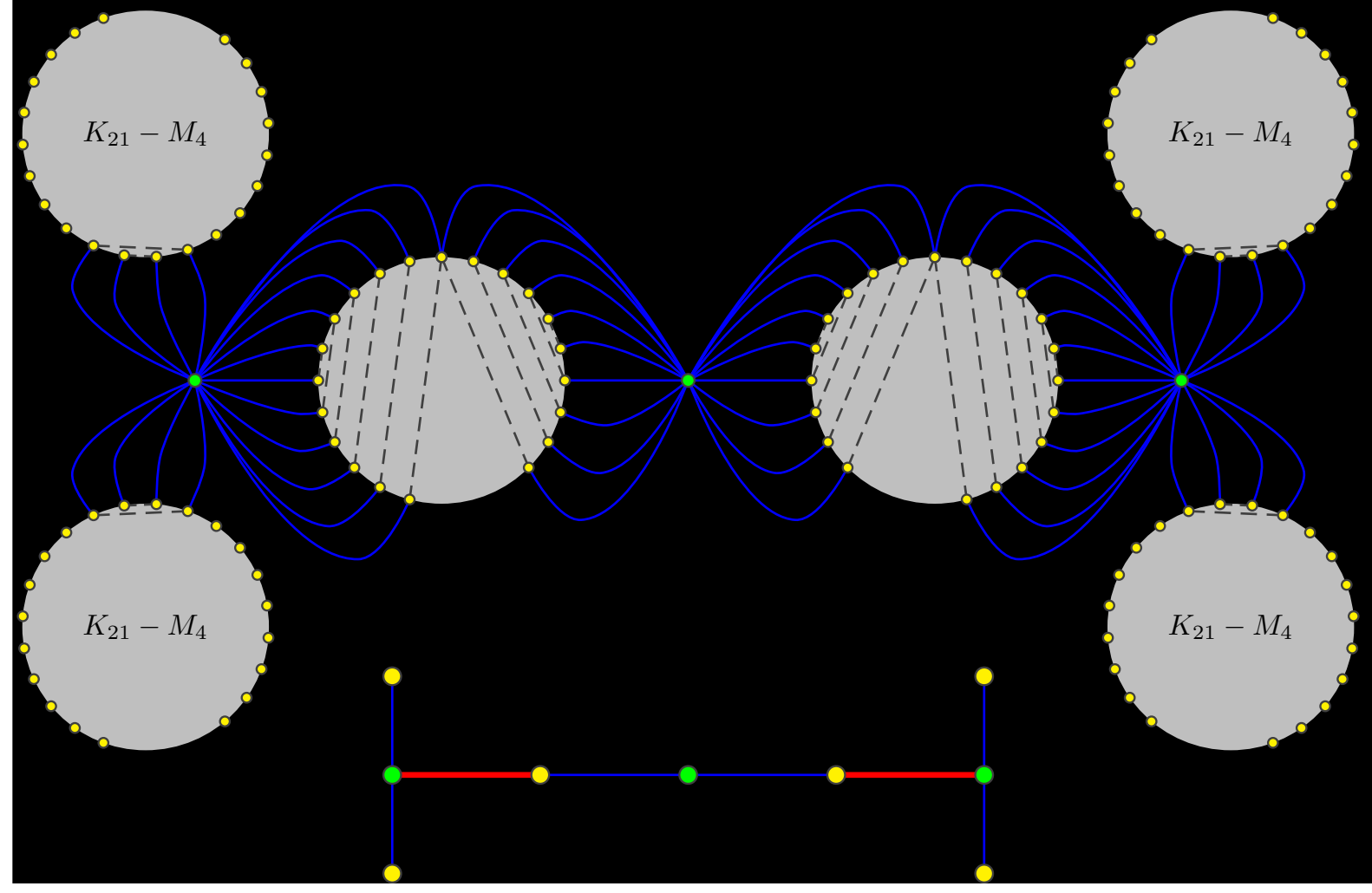

Figure 4: The graph $G$ produced from the given low-high tree with $d=4 \cdot 4+2 \cdot 2=20$. Shaded regions represent cliques minus the dashed matchings.

Since $G$ is connected and $d$-regular, Lemma 2.1 implies that to establish (G4) it suffices to show that every proper contraction minor of $G$ has a vertex of degree less than $d$. Suppose on the contrary that there is a proper contraction minor $G^{\prime}=G / E^{\prime}$ of $G$ with $\delta(G) \geqslant d$. Take such a $G^{\prime}$ with the minimum number of vertices. Thus $G^{\prime}$ has no cut-edges, since contracting a cut-edge does not decrease $\delta$ (since $G^{\prime} \neq K_{2}$ ).

Let $H$ be an arbitrary block of $G$ and consider $H / E^{\prime}$. Suppose that $H / E^{\prime}$ is not contracted to a single vertex. Now $H / E^{\prime} \neq K_{2}$ (as this would either be a nonexistent cut-edge in $G^{\prime}$ or would imply that $G^{\prime}$ has a vertex of degree 1 which is also absurd). But 
if $H / E^{\prime}$ has at least three vertices and $H / E^{\prime}$ is a proper minor of $H$, then by Lemma 7.6, $H / E^{\prime}$ has an inner vertex of degree less than $d$. Hence $H / E^{\prime}$ is either trivial or is left intact in a contraction.

So we may assume that $G^{\prime}$ is obtained by shrinking several blocks of $G$ to single vertices. We may assume that $G^{\prime}$ is obtained by first contracting $k_{i} \geqslant 0$ inner blocks of $G$, and later contracting $k_{e} \geqslant 0$ end-blocks of $G$, where $k_{i}+k_{e} \geqslant 1$. Let $G^{*}$ be the graph obtained after contracting the inner blocks.

Now $k_{i}>0$, as otherwise $G^{\prime}$ is a proper subgraph of $G$. By shrinking $k_{i}$ inner blocks we have reduced the number of cut-vertices by $k_{i}$, and also reduced the sum of their degrees by $k_{i}(d+2)$; see Lemmas 7.2 and 7.3 . Hence $G^{*}$ has at least one cut-vertex $v$ of degree less than $d$, and since $G^{\prime} \neq G^{*}$, at least one contraction of an end-block follows. Finally, contracting an end-block cannot increase $\operatorname{deg}(v)$. This contradiction completes the proof of (G4).

We now prove that minor-minimal minimum-degree graphs can have arbitrary block structure.

Theorem 7.8. For every block decomposition tree $T$, there is an integer $d$ and a graph $G$ such that

(H1) $T$ is the block decomposition tree of $G$,

(H2) $\delta(G) \leqslant 8|E(T)|$, and

(H3) $G \in \widehat{\mathcal{D}}_{d+1}$ where $d=\delta(G)$.

Proof. Let $V_{c} \cup V_{b}$ be the bipartition of $V(T)$, such that every leaf of $T$ is in $V_{b}$. Let $H_{b}$ denote the set of vertices in $V_{b}$ with degree at least 3 in $T$. Thus $T$ is low-high if and only if $H_{b}=\emptyset$. By Theorem 7.7 we may assume that $T$ is not low-high, and $H_{b} \neq \emptyset$. Choose an arbitrary vertex $x \in H_{b}$.

Let $T^{\prime}$ be the tree obtained from $T$ by subdividing each edge that is incident with a vertex in $H_{b}$ once. Each such subdivision vertex and each vertex in $V_{b}-H_{b}$ has degree at most 2 in $T^{\prime}$. Each vertex in $V_{c} \cup H_{b}$ has degree at least 2 in $T^{\prime}$. Thus $T^{\prime}$ is low-high. In particular, $x$ is a high vertex of $T^{\prime}$.

Now $\left|E\left(T^{\prime}\right)\right|<2|E(T)|$ since at least one edge of $T$ is incident with a leaf and did not get subdivided in the construction of $T^{\prime}$. By Theorem 7.7 there exists an integer $d^{\prime} \leqslant 4 E\left(T^{\prime}\right)<8 E(T)$ and a $d^{\prime}$-regular graph $G^{\prime} \in \widehat{\mathcal{D}}_{d^{\prime}+1}$ such that $T^{\prime}$ is the block decomposition tree of $G^{\prime}$. In order to keep the arguments below as simple as possible, assume that $G^{\prime}$ is the graph obtained by the construction in the proof of Theorem 7.7 . Observe that every block of $G^{\prime}$ contains at least 12 vertices, since $T$ has at least one vertex in $H_{b}$. Note that the cut-vertices of $G^{\prime}$ come in two flavours: ones that correspond to vertices of $V_{c}$, and ones that correspond to vertices of $H_{b}$. Similarly, every non-cut-vertex of $G^{\prime}$ corresponds to a vertex of $V_{b}-H_{b}$.

Now define a partition of $V\left(G^{\prime}\right)$ into bags $\left\{B_{y}: y \in V_{b}\right\}$ labelled by vertices $V_{b}$, satisfying the following conditions: 
(C1) for every $y \in H_{b}$ the bag $B_{y}$ contains the cut-vertex $c$ that corresponds to $y$ as well as the interior vertices of every block that contains $c$,

(C2) for every $y \in V_{b}-H_{b}$ the bag $B_{y}$ contains every interior vertex of a block that corresponds to $y \in H_{b}$.

We have so far partitioned every vertex of $G^{\prime}$ that is not a cut-vertex corresponding to a vertex in $V_{c}$.

(C3) if $c$ is a cut-vertex corresponding to a vertex of $V_{c}$, then let $c_{x}$ be its neighbour on some shortest $c-x$ path in $G^{\prime}$, and put $c$ in the bag $B$ that already contains $c_{x}$.

Observe that every block of $G^{\prime}$ contains $d^{\prime}+1$ interior vertices, hence every bag $B_{y}$ contains at least $d^{\prime}+1$ vertices.

Finally we obtain $G$ from $G^{\prime}$ by adding for each bag $B_{y}$ of $G^{\prime}$ a new vertex $\tilde{y}$ which is made adjacent to every vertex of its bag $B_{y}$. Now $G^{\prime}$ is a subgraph of $G$ and every $v \in V\left(G^{\prime}\right)$ has degree equal to $d^{\prime}+1$, and new vertices have degree at least $d^{\prime}+1$. Call this process bag extension and let $d:=d^{\prime}+1$.

Now $G$ contains two types of blocks: small blocks that contain interior vertices of exactly one block of $G^{\prime}$, and big blocks that contain interior vertices of several blocks of $G^{\prime}$. Observe that every big block $B$ contains a separating set of size two comprised of its new vertex and a vertex from $H_{b}$.

Let $B^{\prime}$ (respectively, $B$ ) be an end-block of $G^{\prime}(G)$ and let $c$ be a cut-vertex that separates $B^{\prime}(B)$ from the rest of $G^{\prime}(G)$. By the construction of $G^{\prime}$ there are exactly four edges incident with $c$ whose other end-vertex is in $B^{\prime}(B)$.

Let $e$ be an arbitrary edge of $G$ that is not one of the four edges incident to some cut-vertex of an end-block. Assume that $e$ belongs to block $B$ of $G$. Then there are at least six vertices of degree $d$ in $G$ that are all adjacent to both end-vertices of $e$. This implies that $G / e$ contains at least six vertices of degree less than $d$, and no contraction of an additional two edges of $B$ can eliminate all the vertices of degree less than $d$.

First observe that an end-block of $G$ contains exactly $d+2$ vertices and the other small blocks contain exactly $d+3$ vertices. Every big block on the other hand contains a pair of vertices: the new vertex and a cut-vertex of $G^{\prime}$ corresponding to a vertex in $H_{b}$.

It remains to prove that $G \in \widehat{\mathcal{D}}_{d+1}$. Since every edge has an end-vertex of degree $d$, no edge-deleted subgraph of $G$ has minimum degree at least $d$.

Hence we only have to consider contraction minors of $G$. Let $F \subseteq E(G)$ be a nonempty edge set and let $G^{*}=G / F$. We may split $F=F^{\prime} \cup F^{*}$ so that $F^{\prime} \subseteq E\left(G^{\prime}\right)$.

A block $B$ of $G$ may either get contracted to a single vertex, get partially contracted, or survive the contraction of $F$ without changes.

First assume that $B / F$ gets partially contracted. If $B^{\prime}$ is an end-block, then $B / F$ has exactly $d+1$ vertices obtained by contracting a single edge. This is not possible as a vertex of degree less than $d$ would be created. If $B$ is a small block, then contracting any edge of $B$ leaves at least six vertices of degree less than $d$ in $B$. Since $B$ has $d+3$ vertices in the beginning, an additional two contractions decrease the vertex count below $d+1$, which is absurd. 
Let $B$ be a big block that gets partially contracted. If contraction identifies the new vertex $n$ of $B$ and a cut-vertex $c$ of $G^{\prime}$ corresponding to a vertex in $H_{b}$ then $B / n c$ contains at least six vertices of degree less than $d$ in every block $B^{\prime}$ of $G^{\prime}$ that is a subgraph of $B$. Since $B^{\prime}$ contains $d+2$ vertices, $B^{\prime} / F$ is trivial for every $B^{\prime} \subseteq B$, which is nonsense. Otherwise assume that $B^{\prime} \subseteq B$ is a block of $G^{\prime}$ that gets partially contracted. The $d+1$ interior vertices of $B^{\prime}$ are separated from the rest of $G$ by three vertices. This implies that at most three edges are contracted in order to contract $B^{\prime}$ partially. Yet a single contraction produces six vertices of degree less than $d$ in $B^{\prime}$, so that an additional two contractions do not suffice.

Hence no block of $G$ gets partially contracted in $G / F$. Now $G / F$ may be obtained from $G^{\prime} / F$ by extension of bags, where $G^{\prime} / F$ is a contraction of $G^{\prime}$ that either identifies a block of $G^{\prime}$ or leaves it unchanged. In this case, $G^{\prime} / F$ contains a vertex of degree less than $d^{\prime}$, and bag extension can only increase its degree by one. This completes the proof of Theorem 7.8 .

Open Problem 7.9. By the Robertson-Seymour graph minor theorem, $\widehat{\mathcal{D}}_{k}$ is finite for each fixed $k$. Let $f(k)$ be the maximum number of vertices in a graph in $\widehat{\mathcal{D}}_{k}$. No reasonable bounds on $f(k)$ are known.

- It would be interesting to obtain a simple proof of the finiteness of $\widehat{\mathcal{D}}_{k}$, and to obtain good upper bounds on $f(k)$.

- By Theorem 7.7 with $T=K_{1, s}$, there is a graph $G \in \widehat{\mathcal{D}}_{4 s+1}$ with $1+s(4 s+1)$ vertices. Does every graph in $\widehat{\mathcal{D}}_{k}$ have $O\left(k^{2}\right)$ vertices?

- By Theorem 7.7 with $T=P_{2 s+1}$, there is a graph $G \in \widehat{\mathcal{D}}_{2 s+7}$ with diameter $2 s$. Does every graph in $\mathcal{D}_{k}$ have $O(k)$ diameter?

\section{Complete Multipartite Graphs}

This section characterises the complete multipartite graphs in $\widehat{\mathcal{D}}_{k}$, in $\widehat{\mathcal{C}_{k}}$, in $\widehat{\mathcal{T}}_{k}$, and in $\widehat{\mathcal{P}}_{k}$. See [7, 20, 24] for other results on treewidth obstructions. We first prove three lemmas about complete multipartite graphs. The first says that complete multipartite graphs are highly connected.

Lemma 8.1. Every complete multipartite graph $G$ with minimum degree $k$ is $k$-connected. Moreover, if $v w$ is an edge of $G$ such that both $v$ and $w$ have degree at least $k+1$, then $G-v w$ is k-connected.

Proof. Let $x$ and $y$ be distinct vertices in $G$. It suffices to prove that there is a set of $k$ internally disjoint paths between $x$ and $y$ that avoid $v w$. Let $R$ be the set of vertices coloured differently from both $x$ and $y$.

First suppose that $x$ and $y$ have the same colour. Then $\operatorname{deg}(x)=\operatorname{deg}(y) \geqslant k$, and $P:=\{x r y: r \in R\}$ is a set of $\operatorname{deg}(x)$ internally disjoint paths between $x$ and $y$. If $v w$ is 
in some path in $P$, then without loss of generality $v=x$, implying $\operatorname{deg}(x) \geqslant k+1$, and at least $k$ paths in $P$ avoid $v w$.

Now assume that $x$ and $y$ are coloured differently. Let $S:=\left\{x_{1}, x_{2} \ldots, x_{p}\right\}$ be the colour class that contains $x$, where $x=x_{p}$. Let $T:=\left\{y_{1}, y_{2} \ldots, y_{q}\right\}$ be the colour class that contains $y$, where $y=y_{q}$. Without loss of generality, $n-p=\operatorname{deg}(x) \leqslant \operatorname{deg}(y)=n-q$, implying $q \leqslant p$. Thus

$$
P:=\{x y\} \cup\{x r y: r \in R\} \cup\left\{x y_{i} x_{i} y: i \in[q-1]\right\}
$$

is a set of $\operatorname{deg}(x)$ internally disjoint paths between $x$ and $y$. If $\operatorname{deg}(x) \geqslant k+1$ then at least $k$ paths in $P$ avoid $v w$. Now assume that $v w$ is in some path in $P$, $\operatorname{but} \operatorname{deg}(x)=k$. Since each vertex $x_{i}$ has the same degree as $x$, and $v$ and $w$ both have degree at least $k+1$, the only possibility is that $v=y$ and $w=r$ for some $r \in R$ (or symmetrically $w=y$ and $v=r)$. Thus $\operatorname{deg}(x)<\operatorname{deg}(y)$ and $q<p$. Replace the path $x r y$ in $P$ by the path $x r x_{p-1} y$, which is internally disjoint from the other paths in $P$.

Lemma 8.2. Let $G$ be a complete multipartite graph on $n$ vertices. Then

$$
\kappa(G)=\delta(G)=\operatorname{tw}(G)=\operatorname{pw}(G)=n-\alpha(G) .
$$

Proof. The degree of a vertex $v$ equals $n$ minus the size of the colour class that contains $v$. Since every independent set is contained within a colour class, the size of the largest colour class equals $\alpha(G)$. Thus $\delta(G)=n-\alpha(G)$. We have $\kappa(G) \leqslant \delta(G) \leqslant \operatorname{tw}(G) \leqslant \operatorname{pw}(G)$ for every graph $G$; see [1, 8]. By Lemma 8.1. $\kappa(G) \geqslant \delta(G)$. Thus it suffices to prove that $\delta(G) \geqslant \operatorname{pw}(G)$ for every complete multipartite graph $G$. Let $S=\left\{v_{1}, \ldots, v_{\alpha(G)}\right\}$ be a largest colour class in $G$. Let $X:=V(G)-S$. Observe that $\left(X \cup\left\{v_{1}\right\}, X \cup\right.$ $\left.\left\{v_{2}\right\}, \ldots, X \cup\left\{v_{p}\right\}\right)$ is a path decomposition of $G$ with width $|X|=n-\alpha(G)=\delta(G)$. Thus $\operatorname{pw}(G) \leqslant \delta(G)$.

Lemma 8.3. If $H$ is a minor of a complete multipartite graph $G$, then $H$ can be obtained from $G$ by a sequence of edge contractions, vertex deletions, and edge deletions, such that each operation does not increase the minimum degree, connectivity, treewidth, or pathwidth.

Proof. Every minor of a graph can be obtained by a sequence of edge contractions and vertex deletions, followed by a sequence of edge deletions. Contracting an edge or deleting a vertex in a complete multipartite graph produces another complete multipartite graph. Edge deletions do not increase the minimum degree, connectivity, treewidth, or pathwidth. Thus by Lemma 8.2, it suffices to prove that edge contractions and vertex deletions in complete multipartite graphs do not increase the minimum degree.

Say $G=K_{a_{1}, \ldots, a_{p}}$ has $n$ vertices. Then $G$ has minimum degree $n-\max _{i} a_{i}$. Let $G^{\prime}$ be the graph obtained from $G$ by contracting an edge. Then $G^{\prime}$ is a complete multipartite graph $K_{1, a_{1}^{\prime}, \ldots, a_{p}^{\prime}}$ with $n-1$ vertices, where $a_{i}-1 \leqslant a_{i}^{\prime} \leqslant a_{i}$. Thus

$$
\delta\left(G^{\prime}\right)=n-1-\max _{i} a_{i}^{\prime} \leqslant n-1-\max _{i}\left(a_{i}-1\right)=n-\max _{i} a_{i}=\delta(G) .
$$


Now let $G^{\prime}$ be the graph obtained from $G$ by deleting a vertex. Then $G^{\prime}$ is a complete multipartite graph $K_{a_{1}^{\prime}, \ldots, a_{p}^{\prime}}$ with $n-1$ vertices, where $a_{i}-1 \leqslant a_{i}^{\prime} \leqslant a_{i}$. By the same argument as before, $\delta\left(G^{\prime}\right) \leqslant \delta(G)$.

We now state and prove our first characterisation.

Theorem 8.4. For all $k \geqslant 1$, the following are equivalent for a complete multipartite graph $G$ :

(a) $G \in \widehat{\mathcal{C}_{k}}$

(b) $G \in \widehat{\mathcal{D}}_{k}$

(c) for some $b \geqslant a \geqslant 1$ and $p \geqslant 2$ such that $k+1=a+(p-1) b$,

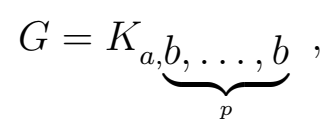

and if $p=2$ then $a=b$.

Proof. (b) $\Longrightarrow$ (a): Say $G \in \widehat{\mathcal{D}}_{k}$. By Lemma 2.1. $\delta(G)=k+1$. By Lemma 8.2. $\kappa(G)=k+1$. By 2 ,,$G \in \widehat{\mathcal{C}}_{k}$.

(a) $\Longrightarrow(\mathrm{c})$ : Consider a complete multipartite graph $G \in \widehat{\mathcal{C}_{k}}$. Thus $\kappa(G) \geqslant k+1$ by Lemma 3.1. If $\kappa(G) \geqslant k+2$ then $\kappa(G-e) \geqslant k+1$ for any edge $e$ of $G$, implying $G \notin \widehat{\mathcal{C}_{k}}$ by Lemma 3.1. Now assume that $\kappa(G)=k+1$. Thus $\delta(G)=k+1$ by Lemma 8.2 .

Suppose on the contrary that adjacent vertices $v$ and $w$ in $G$ both have degree at least $k+2$. By Lemma 8.1, $G-v w$ is $k$-connected, implying $G \notin \widehat{\mathcal{C}}_{k}$. This contradiction proves that no two high-degree vertices in $G$ are adjacent. If two vertices in a complete multipartite graph have distinct degrees, then they are adjacent. Thus the high-degree vertices in $G$ have the same degree, and the vertices of $G$ have at most two distinct degrees. Since the degree of each vertex $v$ equals $|V(G)|$ minus the number of vertices in the colour class that contains $v$, the colour classes of $G$ have at most two distinct sizes. Hence for some $b \geqslant a \geqslant 1$ and $p, q \geqslant 1$,

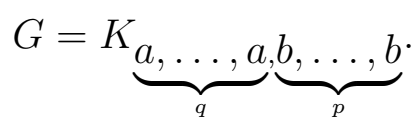

Hence $\kappa(G)=a q+b(p-1)=k+1$. If $a=b$ then, taking $q=1$, we are done. Now assume that $a<b$. Thus $q=1$ as otherwise two high-degree vertices are adjacent. Thus

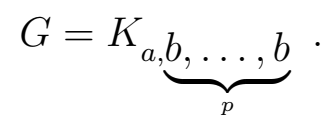

Suppose on the contrary that $p=1$. Then $G=K_{a, b}$ and $\kappa(G)=a$. Contracting one edge in $G$ gives $K_{1, a-1, b-1}$, which by Lemma 8.2 also has connectivity $a$, implying $G \notin \widehat{\mathcal{C}_{k}}$. This contradiction proves that $p \geqslant 2$.

Now suppose that $p=2$. Then $G=K_{a, b, b}$ and $\kappa(G)=a+b$. Contracting one edge gives $K_{1, a, b-1, b-1}$, which by Lemma 8.2 also has connectivity $a+b$ (since $a<b$ ), implying $G \notin \widehat{\mathcal{C}_{k}}$. This contradiction proves that if $p=2$ then $a=b$. 
(c) $\Longrightarrow$ (b) Let

$$
G=K_{a, \underbrace{b, \ldots, b}_{p}}^{b \ldots}
$$

for some $b \geqslant a \geqslant 1$ and $p \geqslant 2$, such that $k+1=a+(p-1) b$ and if $p=2$ then $a=b$. Thus $G$ has minimum degree $k+1$ by Lemma 8.2 . Suppose on the contrary that $G \notin \widehat{\mathcal{D}}_{k}$. By Lemma 3.1, $G$ has a proper minor $H$ with $\delta(H) \geqslant k+1$. By Lemma 8.3, every minor of $G$ in the sequence from $G$ to $H$ has minimum degree at most $k+1$. Thus we can assume that $H$ was obtained from $G$ by a single edge contraction, a vertex deletion, or an edge deletion. In each case we prove that $\delta(H) \leqslant k$, which is the desired contradiction.

First suppose that $H$ is obtained from $G$ by an edge contraction. Then

$$
\text { (i) } H=K_{1, a-1, b-1} \underbrace{b, \ldots, b}_{p-1} \text { or (ii) } H=K_{1, a, b-1, b-1, \underbrace{b, \ldots, b}_{p-2}} \text {. }
$$

In case (i), $\delta(H)=1+(a-1)+(b-1)+(p-2) b=k$. In case (ii) with $p \geqslant 3$, $\delta(H)=1+a+2(b-1)+(p-3) b=k$. Now consider case (ii) with $p=2$. By assumption, $a=b$. Thus $H=K_{1, a, a-1, a-1}$ has minimum degree $1+2(a-1)=k$.

Now suppose that $H$ is obtained from $G$ by a vertex deletion. Then

$$
\text { (i) } H=K_{a-1, \underbrace{b, \ldots, b}_{p}}^{b} \text { or } \quad \text { (ii) } H=K_{a, b-1, \underbrace{b, \ldots, b}_{p-1}} \text {. }
$$

In case (i), $\delta(H)=(a-1)+(p-1) b=k$. In case (ii), $\delta(H)=a+(b-1)+(p-2) b=k$ (since $p \geqslant 2$ ).

In $G$, every edge is incident to a vertex of degree $k+1$. Thus, if $H$ is obtained from $G$ by an edge deletion, then $\delta(H) \leqslant k$.

The remainder of this section is devoted to characterising the complete multipartite graphs in $\widehat{\mathcal{T}}_{k}$ and in $\widehat{\mathcal{P}}_{k}$. We start with a lemma about independent sets in complete multipartite graphs.

Lemma 8.5. For every edge vw in a complete multipartite graph $G$, every independent set in $G-v w$ is either $\{v, w\}$ or is also independent in $G$. Thus if $\alpha(G) \geqslant 2$ (that is, $G$ is not a complete graph) then $\alpha(G-v w)=\alpha(G)$.

Proof. Let $G^{\prime}:=G-v w$. Let $I$ be an independent set in $G^{\prime}$ that is not independent in $G$. Thus both $v$ and $w$ are in $I$. Let $S$ be the colour class containing $v$. Every vertex not in $S \cup\{w\}$ is adjacent to $v$ in $G^{\prime}$. Thus $I \subseteq S \cup\{w\}$. Every vertex in $S-\{v\}$ is adjacent to $w$ in $G^{\prime}$. Thus $I:=\{v, w\}$. Hence every independent set in $G^{\prime}$ is either $\{v, w\}$ or is also independent in $G$. Thus $\alpha\left(G^{\prime}\right)=\alpha(G)$ whenever $\alpha(G) \geqslant 2$.

To prove lower bounds on treewidth we use the following idea. Let $G$ be a graph. Two subgraphs $X$ and $Y$ of $G$ touch if $X \cap Y \neq \emptyset$ or there is an edge of $G$ between $X$ and $Y$. A bramble in $G$ is a set of pairwise touching connected subgraphs. The subgraphs are called bramble elements. A set $S$ of vertices in $G$ is a hitting set of a bramble $\mathcal{B}$ if $S$ 
intersects every element of $\mathcal{B}$. The order of $\mathcal{B}$ is the minimum size of a hitting set. The following 'Treewidth Duality Theorem' shows the intimate relationship between treewidth and brambles.

Theorem 8.6 (Seymour and Thomas [26]). A graph $G$ has treewidth at least $k$ if and only if $G$ contains a bramble of order at least $k+1$.

For example, say $G$ is a complete multipartite graph on $n$ vertices. Let $S$ be a set of vertices in $G$, one from each colour class; that is, $S$ is a maximum clique in $G$. Then it is easily seen that $\mathcal{B}:=E(G) \cup S$ is a bramble of order $n-\alpha(G)+1$, and thus $\operatorname{tw}(G) \geqslant n-\alpha(G)$ by Theorem 8.6 (confirming Lemma 8.2). The next two lemmas give circumstances when an edge can be deleted from a complete multipartite graph without decreasing the treewidth.

Lemma 8.7. Let $G$ be a complete multipartite graph with $\alpha(G) \geqslant 3$, such that at least two colour classes contain at least two vertices. Let $v w$ be an edge, where both $v$ and $w$ are in colour classes that contain at least two vertices. Then $\operatorname{tw}(G-v w)=\operatorname{tw}(G)$.

Proof. Say $G$ has $n$ vertices. Let $G^{\prime}:=G-v w$. By Lemmas 8.2 and 8.5, $\operatorname{tw}(G)=$ $n-\alpha(G)=n-\alpha\left(G^{\prime}\right)$. Clearly $\operatorname{tw}\left(G^{\prime}\right) \leqslant \operatorname{tw}(G)$. Thus it suffices to prove that $\operatorname{tw}\left(G^{\prime}\right) \geqslant$ $n-\alpha\left(G^{\prime}\right)$.

Since $v$ and $w$ are in colour classes that contain at least two vertices, there is a set $S$ of vertices, such that both $v$ and $w$ are not in $S$, and each colour class has exactly one vertex in $S$. Thus $S$ is a maximum clique in $G$ and in $G^{\prime}$. Let $\mathcal{B}:=E\left(G^{\prime}\right) \cup S$.

We now prove that $\mathcal{B}$ is a bramble in $G^{\prime}$. Each element of $\mathcal{B}$ induces a connected subgraph in $G^{\prime}$. Every pair of vertices in $S$ are adjacent. Say $x \in S$ and $p q \in E\left(G^{\prime}\right)$. Since $p$ and $q$ have distinct colours, $x$ is coloured differently from $p$ or $q$, and thus $x$ is adjacent to $p$ or $q$ (since $x \neq v$ and $x \neq w$ ). Hence $x$ touches $p q$. Say $p q \in E\left(G^{\prime}\right)$ and $r s \in E\left(G^{\prime}\right)$. If $\{p, q\} \cap\{r, s\} \neq \emptyset$ then $p q$ and $r s$ touch. So assume that $p, q, r, s$ are distinct. Thus there are at least two edges in $G$ between $\{p, q\}$ and $\{r, s\}$, one of which is not $v w$. Hence $p q$ touches $r s$. Therefore $\mathcal{B}$ is a bramble in $G^{\prime}$.

Let $H$ be a minimum hitting set of $\mathcal{B}$. If $|H| \geqslant n-\alpha\left(G^{\prime}\right)+1$, then $\mathcal{B}$ has order at least $n-\alpha\left(G^{\prime}\right)+1$, implying $\operatorname{tw}\left(G^{\prime}\right) \geqslant n-\alpha\left(G^{\prime}\right)$ by Theorem 8.6, and we are done. Now assume that $|H| \leqslant n-\alpha\left(G^{\prime}\right)$.

Since every edge of $G^{\prime}$ is in $\mathcal{B}, H$ is a vertex cover of $G^{\prime}$, and $V\left(G^{\prime}\right)-H$ is an independent set of $G^{\prime}$. Thus $n-|H| \leqslant \alpha\left(G^{\prime}\right)$. Hence $|H|=n-\alpha\left(G^{\prime}\right)$, and $V\left(G^{\prime}\right)-H$ is a maximum independent set of $G^{\prime}$. By Lemma 8.5, every independent set of $G^{\prime}$ is $\{v, w\}$ or is an independent set of $G$. Since $\alpha\left(G^{\prime}\right) \geqslant 3,\{v, w\}$ is not a maximum independent set. Hence $V(G)-H$ is a maximum independent set of $G$. That is, $V(G)-H$ is a colour class in $G$, which implies that $H$ does not contain one vertex in $S$, and $H$ is not a hitting set of $\mathcal{B}$. This is the desired contradiction.

Lemma 8.8. Let $G$ be a complete multipartite graph with $\alpha(G) \geqslant 2$, and at least one singleton colour class. Let $v w$ be an edge, where $v$ is in a singleton colour class, and $w$ is in a colour classes that contains at least two vertices. Then $\operatorname{tw}(G-v w)=\operatorname{tw}(G)$. 
Proof. Say $G$ has $n$ vertices. Let $G^{\prime}:=G-v w$. By Lemmas 8.2 and 8.5, $\operatorname{tw}(G)=$ $n-\alpha(G)=n-\alpha\left(G^{\prime}\right)$. Clearly $\operatorname{tw}\left(G^{\prime}\right) \leqslant \operatorname{tw}(G)$. Thus it suffices to prove that $\operatorname{tw}\left(G^{\prime}\right) \geqslant$ $n-\alpha\left(G^{\prime}\right)$.

By assumption, there is a set $S$ of vertices, such that $w \notin S$, and every colour class has exactly one vertex in $S$. Thus $v \in S$. Note that $S$ is a maximum clique in $G$ and in $G^{\prime}$. Let $\mathcal{B}:=E\left(G^{\prime}\right) \cup S$.

We now prove that $\mathcal{B}$ is a bramble in $G^{\prime}$. Each element of $\mathcal{B}$ induces a connected subgraph in $G^{\prime}$. Every pair of vertices in $S$ are adjacent. Consider $v \in S$ and $p q \in E\left(G^{\prime}\right)$. Since $v$ is in a singleton colour class, $v$ is adjacent to both $p$ and $q$ in $G$, and thus $v$ is adjacent to $p$ or $q$ in $G^{\prime}$. Hence $v$ touches $p q$. Now consider $x \in S-\{v\}$ and $p q \in E\left(G^{\prime}\right)$. Since $p$ and $q$ have distinct colours, $x$ is coloured differently from $p$ or $q$, and thus $x$ is adjacent to $p$ or $q$ (since $x \neq v$ and $x \neq w$ ). Hence $x$ touches $p q$. Finally consider two edges $p q \in E\left(G^{\prime}\right)$ and $r s \in E\left(G^{\prime}\right)$. If $\{p, q\} \cap\{r, s\} \neq \emptyset$ then $p q$ and $r s$ touch. So assume that $p, q, r, s$ are distinct. Thus there are at least two edges in $G$ between $\{p, q\}$ and $\{r, s\}$, one of which is not $v w$. Hence $p q$ touches $r s$. Therefore $\mathcal{B}$ is a bramble in $G^{\prime}$.

Let $H$ be a minimum hitting set of $\mathcal{B}$. If $|H| \geqslant n-\alpha\left(G^{\prime}\right)+1$, then $\mathcal{B}$ has order at least $n-\alpha\left(G^{\prime}\right)+1$, implying $\operatorname{tw}\left(G^{\prime}\right) \geqslant n-\alpha\left(G^{\prime}\right)$ by Theorem 8.6, and we are done. Now assume that $|H| \leqslant n-\alpha\left(G^{\prime}\right)$.

Since every edge of $G^{\prime}$ is in $\mathcal{B}, H$ is a vertex cover of $G^{\prime}$, and $V\left(G^{\prime}\right)-H$ is an independent set of $G^{\prime}$. Thus $n-|H| \leqslant \alpha\left(G^{\prime}\right)$. Hence $|H|=n-\alpha\left(G^{\prime}\right)$, and $V\left(G^{\prime}\right)-H$ is a maximum independent set of $G^{\prime}$. By Lemma 8.5. every independent set of $G^{\prime}$ is $\{v, w\}$ or is an independent set of $G$. If $V\left(G^{\prime}\right)-H=\{v, w\}$ then $H$ does not contain $v$, and $H$ is not a hitting set of $\mathcal{B}$, which is a contradiction. Otherwise, $V(G)-H$ is a maximum independent set of $G$. That is, $V(G)-H$ is a colour class in $G$, which implies that $H$ does not contain some vertex in $S$, and $H$ is not a hitting set of $\mathcal{B}$. This is the desired contradiction.

Theorem 8.9. For all $k \geqslant 1$, the following are equivalent for a complete multipartite graph $G$ :
(a) $G \in \widehat{\mathcal{T}}_{k}$
(b) $G \in \widehat{\mathcal{P}}_{k}$
(c) $G=K_{k+2}$, or $k \geqslant 3$ is odd and $G=K_{\underbrace{2, \ldots, 2}_{(k+3) / 2}}$.

Proof. (b) $\Longrightarrow$ (a): Say $G \in \widehat{\mathcal{P}}_{k}$. By Lemma 3.1. $\operatorname{pw}(G)=k+1$. By Lemma 8.2, $\operatorname{tw}(G)=k+1$. By (4), $G \in \widehat{\mathcal{T}}_{k}$.

(a) $\Longrightarrow(\mathrm{c})$ : Say $G \in \widehat{\mathcal{T}}_{k}$. By Lemma $3.1, \operatorname{tw}(G) \geqslant k+1$. If $\operatorname{tw}(G) \geqslant k+2$ then $\operatorname{tw}(G-v) \geqslant k+1$ for any vertex $v$ of $G$, implying $G \notin \widehat{\mathcal{T}}_{k}$ by Lemma 3.1. Now assume that $\operatorname{tw}(G)=k+1$. Thus $\delta(G)=k+1$ by Lemma 8.2 , and $G \in \widehat{\mathcal{D}}_{k}$ by (3). By Theorem 8.4 .

$$
G=K_{a, \underbrace{b, \ldots, b}_{p},}^{, \ldots}
$$

for some $b \geqslant a \geqslant 1$ and $p \geqslant 2$, such that $k+1=a+(p-1) b$ and if $p=2$ then $a=b$. 
Case. $\boldsymbol{b}=1$ : Then $a=1$ and $G=K_{k+2}$, and we are done.

Case. $\boldsymbol{b}=2$ : Then $k+3=a+2 p$. If $a=1$, then by Lemma 8.8, $\operatorname{tw}(G-e)=\operatorname{tw}(G)$ for some edge $e$ of $G$, implying $G \notin \mathcal{T}_{k}$ by Lemma 3.1. Otherwise $a=2$. Thus $k=2 p-1$ is odd, and $k \geqslant 3$ since $p \geqslant 2$. Hence

$$
G=K_{(k+3) / 2}^{2, \ldots, 2} .
$$

Case. $\boldsymbol{b} \geqslant 3$ : Then $\alpha(G) \geqslant 3$. Since $p \geqslant 2$, there are at least two colour class that contain at least two vertices, and by Lemma 8.7, $\operatorname{tw}(G-e)=\operatorname{tw}(G)$ for some edge $e$ of $G$, implying $G \notin \mathcal{T}_{k}$ by Lemma 3.1 .

(c) $\Longrightarrow$ (b): If $G=K_{k+2}$ then $G \in \widehat{\mathcal{P}}_{k}$ by Lemma 3.1. Now assume that $k \geqslant 3$ is odd and

$$
G=K_{(k+3) / 2}^{2, \ldots, 2} .
$$

Thus $\operatorname{pw}(G)=k+1$ by Lemma 8.2 . Suppose on the contrary that $G \notin \widehat{\mathcal{P}}_{k}$. By Lemma 3.1 , $G$ has a proper minor $H$ with $\mathrm{pw}(H) \geqslant k+1$. By Lemma 8.3, every minor of $G$ in the sequence from $G$ to $H$ has pathwidth at most $k+1$. Thus we can assume that $H$ was obtained from $G$ by a single edge contraction, a vertex deletion, or an edge deletion. Since an edge contraction or a vertex deletion produce another complete multipartite graph, and the minimum degree of a complete multipartite graph equals its pathwidth (Lemma 8.2), the same proof used in Theorem 8.4 shows that $\mathrm{pw}(H) \leqslant k$. Now assume that $H=G-v w$ for some edge $v w$ of $G$. Let $x$ be the other vertex in the colour class that contains $v$. Let $y$ be the other vertex in the colour class that contains $w$. Let $S:=V(G)-\{v, w, x, y\}$. Then $(S \cup\{v, y\}, S \cup\{x, y\}, S \cup\{x, w\})$ is a path decomposition of $H$ with width $k$, which is the desired contradiction.

Open Problem 8.10. Complete multipartite graphs have diameter 2. Are there generalisations of Theorems 8.4 and 8.9 for all diameter-2 graphs in $\widehat{\mathcal{D}}_{k}$ or in $\widehat{\mathcal{T}}_{k}$ ?

\section{Acknowledgements}

Thanks to Vida Dujmović who first proved Lemma 6.5.

\section{References}

[1] Hans L. Bodlaender. A partial $k$-arboretum of graphs with bounded treewidth. Theoret. Comput. Sci., 209(1-2):1-45, 1998. doi:10.1016/S0304-3975(97) 00228-4.

[2] Hans L. Bodlaender and Thomas Wolle. Contraction degeneracy on cographs. Tech. Rep. UU-CS-2004-031, Department of Information and Computing Sciences, Utrecht University, 2004. http://www.cs.uu.nl/research/techreps/ UU-CS-2004-031.html. 
[3] Hans L. Bodlaender, Thomas Wolle, and Arie M. C. A. Koster. Contraction and treewidth lower bounds. J. Graph Algorithms Appl., 10(1):5-49, 2006. http://jgaa.info/accepted/2006/BodlaenderWolleKoster2006.10.1.pdf.

[4] Drago Bokal, Éva Czabarka, László A. Székely, and Imrich VrT̃o. Graph minors and the crossing number of graphs. Electron. Notes Discrete Math., 28:169-175, 2007. doi:10.1016/j.endm.2007.01.024.

[5] Drago Bokal, Gašper Fijavž, and Bojan Mohar. The minor crossing number. SIAM J. Discrete Math., 20(2):344-356, 2006. doi:10.1137/05062706X.

[6] Drago Bokal, Gašper Fijavž, and David R. Wood. The minor crossing number of graphs with an excluded minor. Electron. J. Combin., 15(R4), 2008. http://www. combinatorics.org/Volume_15/Abstracts/v15i1r4.html.

[7] Janka Chlebíková. The structure of obstructions to treewidth and pathwidth. Discrete Appl. Math., 120(1-3):61-71, 2002. doi:10.1016/S0166-218X(01)00281-5.

[8] Reinhard Diestel. Graph theory, vol. 173 of Graduate Texts in Mathematics. Springer, 2nd edn., 2000. http://diestel-graph-theory.com/index.html.

[9] Gabriel A. Dirac. A property of 4-chromatic graphs and some remarks on critical graphs. J. London Math. Soc., 27:85-92, 1952. doi:10.1112/jlms/s1-27.1.85.

[10] Gabriel A. Dirac. On the four-colour conjecture. Proc. London Math. Soc. (3), 13:193-218, 1963.

[11] GaŠPer FiJavž. Graphs Minors and Connectivity. Ph.D. thesis, Univerity of Ljubljana, Slovenia, 2001.

[12] GaŠPER FiJAVž. Minor-minimal 5-connected projective planar graphs, 2002. Submitted.

[13] GaŠPER FiJAVž. Minor-minimal 6-regular graphs in the Klein bottle. European J. Combin., 25(6):893-898, 2004. doi:10.1016/j.ejc.2003.09.021.

[14] Gašper Fijavž. Contractions of 6-connected toroidal graphs. J. Combin. Theory Ser. B, 97(4):553-570, 2007. doi:10.1016/j.jctb.2006.09.003.

[15] Gašper Fijavž and Bojan Mohar. $K_{6}$-minors in projective planar graphs. Combinatorica, 23(3):453-465, 2003. doi:10.1007/s00493-003-0027-y.

[16] Hugo Hadwiger. Über eine Klassifikation der Streckenkomplexe. Vierteljschr. Naturforsch. Ges. Zürich, 88:133-142, 1943.

[17] Rudolf Halin and Heinz A. Jung. Über Minimalstrukturen von Graphen, insbesondere von $n$-fach zusammenhängenden Graphen. Math. Ann., 152:75-94, 1963. 
[18] Arie M. C. A. Koster, Thomas Wolle, and Hans L. Bodlaender. Degree-based treewidth lower bounds. In SotiRis E. Nikoletseas, ed., Proc. 4 th International Workshop on Experimental and Efficient Algorithms (WEA '05), vol. 3503 of Lecture Notes in Computer Science, pp. 101-112. Springer, 2005. doi:10.1007/11427186_11.

[19] Matthias Kriesell. A survey on contractible edges in graphs of a prescribed vertex connectivity. Graphs Combin., 18(1):1-30, 2002. doi:10.1007/s003730200000.

[20] Brian Lucena. Achievable sets, brambles, and sparse treewidth obstructions. Discrete Appl. Math., 155(8):1055-1065, 2007. doi:10.1016/j.dam.2006.11.006.

[21] Wolfgang Mader. Homomorphiesätze für Graphen. Math. Ann., 178:154-168, 1968.

[22] Wolfgang Mader. High connectivity keeping sets in graphs and digraphs. Discrete Math., 302(1-3):173-187, 2005. doi:10.1016/j.disc.2004.07.032.

[23] John Maharry. An excluded minor theorem for the octahedron. J. Graph Theory, 31(2):95-100, 1999. doi:10.1002/jgt.20272.

[24] Siddharthan Ramachandramurthi. The structure and number of obstructions to treewidth. SIAM J. Discrete Math., 10(1):146-157, 1997. doi:10.1137/S0895480195280010.

[25] Neil Robertson and Paul D. Seymour. Graph minors I-XXIII. J. Combin. Theory Ser. B, 1983-2010.

[26] Paul D. Seymour and Robin Thomas. Graph searching and a min$\max$ theorem for tree-width. J. Combin. Theory Ser. B, 58(1):22-33, 1993. doi:10.1006/jctb.1993.1027.

[27] William T. Tutte. A theory of 3-connected graphs. Nederl. Akad. Wetensch. Proc. Ser. A $64=$ Indag. Math., 23:441-455, 1961.

[28] William T. Tutte. How to draw a graph. Proc. London Math. Soc., 13(3):743-768, 1963.

[29] Klaus Wagner. Über eine Eigenschaft der ebene Komplexe. Math. Ann., 114:570 590, 1937. doi:10.1007/BF01594196.

[30] Hassler Whitney. 2-Isomorphic Graphs. Amer. J. Math., 55(1-4):245-254, 1933.

[31] Thomas Wolle and Hans L. Bodlaender. A note on edge contraction. Tech. Rep. UU-CS-2004-028, Department of Information and Computing Sciences, Utrecht University, 2004. http://www.cs.uu.nl/research/techreps/UU-CS-2004-028. html. 
[32] Thomas Wolle, Arie M. C. A. Koster, and Hans L. Bodlaender. A note on contraction degeneracy. Tech. Rep. UU-CS-2004-042, Department of Information and Computing Sciences, Utrecht University, 2004. http://www.cs.uu.nl/ research/techreps/UU-CS-2004-042.html.

[33] Douglas R. Woodall. A short proof of a theorem of Dirac's about Hadwiger's conjecture. J. Graph Theory, 16(1):79-80, 1992. doi:10.1002/jgt.3190160109.

[34] Bernhardine Zeidl. Über 4- und 5-chrome Graphen. Monatsh. Math., 62:212-218, 1958.

\section{A Graphs with Minimum Degree Four}

In this appendix we prove the following result.

Theorem A.1. Every graph with minimum degree at least 4 contain a 4-connected minor.

The following stronger result enables an inductive proof of Theorem A.1.

Lemma A.2. Let $G$ be a graph with at least 5 vertices, such that the vertices of degree at most 3 induce a clique. Then $G$ contains a 4-connected minor.

Proof. Let $G$ be a counterexample with the minimum number of vertices. Let $K=$ $\left\{v_{1}, \ldots, v_{|K|}\right\}$ denote the (possibly empty) clique of vertices of degree at most 3.

In each case below we exhibit a proper minor $G^{\prime}$ of $G$, for which it is easy to verify that the vertices of degree at most 3 induce a clique. Moreover, $\left|V\left(G^{\prime}\right)\right| \geqslant 5$ since there is a vertex of degree at least 4 in $G$, whose degree does not decrease in $G^{\prime}$. Thus $G^{\prime}$ satisfies the conditions of the lemma, which contradicts the minimality of $G$.

If $e$ is an edge incident to a vertex of degree at most 2, then let $G^{\prime}:=G / e$. Now assume that $\delta(G) \geqslant 3$.

Let $S$ be a minimal separator in $G$, and let $\left\{G_{1}, G_{2}\right\}$ be the corresponding separation, so that $S=V\left(G_{1} \cap G_{2}\right)$. Without loss of generality, $K \subseteq G_{1}$. If $|S|=1$, then let $G^{\prime}:=G_{2}$. If $|S|=2$, say $S=\left\{s_{1}, s_{2}\right\}$, then there exists an $s_{1}-s_{2}$ path in $G_{1}$, and so $G^{\prime}:=G_{2}+s_{1} s_{2}$ is the desired minor.

Thus $G$ is 3-connected and each vertex in $K$ has degree 3. Let $N(K)$ denote the subgraph induced by the neighbours of $K$.

First suppose that $|K|=2$. Both $v_{1}$ and $v_{2}$ have at least two neighbours in $N(K)$. If $|N(K)| \geqslant 3$, then at most one vertex is adjacent to both $v_{1}$ and $v_{2}$. Let $G^{\prime}:=G / v_{1} v_{2}$. If, on the other hand, $N(K)=\left\{u_{1}, u_{2}\right\}$, then let $G^{\prime}$ be obtained from $G$ by contracting the triangle $v_{1} v_{2} u_{1}$. Since $G$ has a vertex of degree at least 4 other than $u_{1}, u_{2}$, so does $G^{\prime}$.

If $|K|=1$ or $|K|=3$ then $|N(K)|=3$. Every vertex of $N(K)$ is adjacent to exactly one vertex of $K$, so $G$ has more than $|K|+|N(K)|$ vertices. If $N(K)$ induces a clique, then let $G^{\prime}:=G-V(K)$. Otherwise let $u_{1}$ be a vertex whose degree in $N(K)$ is as 
small as possible (is at most 1 ) and let $v_{1}$ be its unique neighbour in $K$. In this case, let $G^{\prime}:=G / u_{1} v_{1}$. Therefore we may assume that $G$ is 3 -connected with $\delta(G) \geqslant 4$.

Suppose that $G$ contains a 3 -separation $\left\{G_{1}, G_{2}\right\}$ with separator $S=\left\{s_{1}, s_{2}, s_{3}\right\}=$ $V\left(G_{1} \cap G_{2}\right)$. Consider the subgraph $G_{1}$. Each vertex in $S$ has degree at least 1 in $G_{1}$. Now $V\left(G_{1}-S\right) \neq \emptyset$ and every vertex in $G_{1}-S$ has degree at least 4 in $G_{1}$. A forest that contains a vertex of degree at least 4 has at least 4 leaves. Thus $G_{1}$ is not a forest. Hence $G_{1}$ contains a cycle $C$. By Menger's Theorem, there are three disjoint $C-S$ paths in $G_{1}$. By contracting $C$ together with these three paths to a triangle on $S$, observe that $G^{\prime}:=G_{2}+s_{1} s_{2}+s_{1} s_{3}+s_{2} s_{3}$ is the desired minor. Hence $G$ has no 3-separation and is thus 4-connected.

For completeness we include a proof of the following theorem of Halin and Jung [17] based on classical results by Wagner, Whitney and Tutte.

Theorem A.3 ([17]). Every 4-connected graph contains $K_{5}$ or $K_{2,2,2}$ as a minor.

Proof. Suppose that $G$ is 4 -connected and has no $K_{5}$-minor. Thus $G$ is planar by Wagner's characterisation of graphs with no $K_{5}$-minor [29]. Fix a plane embedding of $G$. Let $v$ be any vertex of $G$. Let $w_{1}, w_{2}, w_{3}, w_{4}$ be four of the neighbours of $v$ in cyclic order around $v$. Let $C$ be the facial cycle in the induced plane embedding of $G-v$, such that the interior of $C$ contains $v$. Whitney [30] proved that every 3-connected planar graph has a unique plane embedding. Moreover, Tutte 28] proved that the faces of this embedding are exactly the induced nonseparating cycles. Since $G-v$ is 3 -connected, each face in the induced plane embedding of $G-v$ is an induced nonseparating cycle. In particular, $C$ is induced and nonseparating in $G-v$. Since $C$ is separating, $(G-v)-C$ is connected. Since $C$ is induced, each vertex $w_{i}$ has exactly two neighbours in $C$, and at least one neighbour in $(G-v)-C$. Hence, contracting $(G-v)-C$ to a single vertex, and contracting $C$ to the 4-cycle $\left(w_{1}, w_{2}, w_{3}, w_{4}\right)$ produces a $K_{2,2,2}$-minor in $G$.

Note that Theorem A.3 can also be concluded from a theorem of Maharry [23], who proved that every 4-connected graph with no $K_{2,2,2}$ minor is isomorphic to the square of an odd cycle, which is easily seen to contain a $K_{5}$-minor. Theorems A.1 and A.3 imply:

Corollary A.4. Every graph with minimum degree at least 4 contains $K_{5}$ or $K_{2,2,2}$ as a minor. 